\title{
Estudio micológico de El Canal y Los Tiles (La Palma, Islas Canarias). V. Datos adicionales
}

\author{
por \\ Esperanza Beltrán Tejera ${ }^{1}$, Luis Quijada, Jonathan Díaz, J. Laura Rodríguez-Armas, Ángel Bañares \& Julio Leal \\ Departamento de Biología Vegetal (Botánica), Universidad de La Laguna, E-38071 La Laguna, España \\ ebeltran@ull.es ${ }^{1}$
}

\begin{abstract}
Resumen
Beltrán Tejera, E., Quijada L., Díaz, J., Rodríguez-Armas, J.L., Bañares, A. \& Leal, J. 2009. Estudio micológico de El Canal y Los Tiles (La Palma, Islas Canarias). V. Datos adicionales. Anales Jard. Bot. Madrid 66S1: 93-107.

Se presenta un estudio sobre 66 especies, pertenecientes a las divisiones Myxomycota (21), Ascomycota (29) y Basidiomycota (16), encontradas en la antigua Reserva de la Biosfera El Canal y Los Tiles. De ellas 11 se citan por primera vez para la isla de La Palma, siendo 5 de éstas nuevas para Canarias. Se hacen comentarios taxonómicos sobre algunos táxones conflictivos, además de amplia información sobre su distribución en la Región Macaronésica. En base a publicaciones propias anteriores, se aportan datos globales sobre biodiversidad, sustratos y distribución de la micobiota en las diferentes unidades ambientales presentes en el área de estudio.
\end{abstract}

Palabras clave: hongos, Myxomycota, diversidad, taxonomía, corología, laurisilva.

\section{Introducción}

El 6 de noviembre de 2002, la isla de La Palma en su totalidad fue declarada Reserva Mundial de la Biosfera por la UNESCO, ampliando así sus límites, circunscritos hasta entonces al paraje de El Canal y Los Tiles, comúnmente denominado Los Tiles, topónimo que alude al nombre común (Til) de Ocotea foetens (Aiton) Baill., una de las especies de Lauraceae más abundantes de esta localidad, ubicada en el Parque Natural de las Nieves, perteneciente a la red canaria de espacios naturales protegidos (www.lapalmabiosfera.com).

Nuestras investigaciones micológicas en El Canal y Los Tiles comenzaron en 1987 y fue una parte de un proyecto más amplio que abarcó el inventario integral de la biota no vascular terrestre de la zona. Hasta el

\begin{abstract}
Beltrán Tejera, E., Quijada L., Díaz, J., Rodríguez-Armas, J.L., Bañares, A. \& Leal, J. 2009. Mycological study of the El Canal y Los Tiles (La Palma, Canary Islands). V. Additional data. Anales Jard. Bot. Madrid 6651: 93-107 (in Spanish).

An annotated catalogue of 66 taxa, collected in the early MAB Reserve El Canal y Los Tiles is presented. Among these taxa, 11 species are new for La Palma island and 5 are recorded for the first time in the Canary Islands. Taxonomic comments on some critical species and information about the distribution in the Macaronesian bioregion of all the studied taxa are given. Based on our previous publications, global data on biodiversity, substrates and distribution of the mycobiota in the different plant communities present in the sampled area are analyzed.
\end{abstract}

Keywords: fungi, Myxomycota, diversity, taxonomy, chorology, laurel forest.

momento y en relación con la micobiota, se han publicado los resultados sobre los Aphyllophorales (Rodríguez-Armas \& al., 1992a,b, 1994; Larsen \& al., 1994; Rodríguez-Armas \& Beltrán Tejera, 1995); Agaricales (Bañares \& al., 1992, 1994; Esteve-Raventós \& al., 1998; Bañares \& Arnolds, 2002); y Xylariales p.p. (Stadler \& al., 2008), en los que se han dado a conocer 209 táxones, de los que 96 se citaron por primera vez para La Palma y de éstos 33 fueron novedades para el Archipiélago Canario. El valor micológico de la antigua Reserva se ha visto acrecentado por la descripción de tres nuevas especies para la ciencia: Tomentella oligofibula Larsen, Beltrán-Tej. \& Rodríguez-Armas (Larsen \& al., 1994), Hygrocybe monteverdae Bañares \& Arnolds (Bañares \& Arnolds, 2002), Hypoxylon canariense J. Fournier, M. Stadler, Beltrán-Tej. \& 
Granmo (Stadler \& al., 2008), y una variedad: Marasmius epiphylloides var. latispora Bañares \& BeltránTej. (Bañares \& al., 1994).

En este trabajo damos a conocer los resultados obtenidos del estudio de las especies de Myxomycota, Ascomycota y Basidiomycota p.p. (Ustilaginomycetidae, Tremellomycetidae, Agaricomycetidae p.p.).

\section{Área de estudio}

El Paraje Natural de El Canal y Los Tiles se localiza en el noreste de la isla, pertenece al término municipal de San Andrés y Sauces, isla de La Palma, Provincia de Santa Cruz de Tenerife (Islas Canarias, España). Posee una superficie de 511,300 ha, comprende casi la totalidad del Barranco del Agua y su cabecera, en la que se unen los de Marcos y Cordero. Su cota más alta es la Cruz de Vázquez (1475 m), al SW, y la inferior, La Tomada (250 m), al NE (Fig. 1).

\section{Vegetación de la zona de estudio}

La mayor parte de la superficie de los antiguos límites de la Reserva se halla ocupada por una de las mejores representaciones del bosque de laurisilva s.l. de la Región Macaronésica. La abundancia de agua, que discurre la mayor parte del año por el cauce, saltos y paredones, propicia una vegetación exuberante de tipo subtropical, caracterizada en los fondos de barranco por el típico bosque de galería, que lleva asociada una notable representación criptogámica, constituyendo en ocasiones ricas comunidades hidrohigrófilas, tanto rupícolas como reófilas en sentido amplio. Los fondos de barranco, más sombríos y con mayor humedad edáfica, se hallan ocupados por una laurisilva húmeda, donde son sobre todo abundantes Ocotea foetens y Laurus novocanariensis Rivas-Martínez \& al., en el estrato arbóreo. En estos ambientes las comunidades briofíticas, sobre diferentes sustratos, así como los helechos, tienen un elevado protagonismo, alcanzando en este último caso coberturas próximas al $90 \%$ y siendo particularmente abundantes $D i$ plazium caudatum (Cav.) Jermy y Woodwardia radicans (L.) Sm. Según la pendiente y orientación de las laderas que caen al cauce, esta comunidad higroumbrófila se va sustituyendo en los primeros tramos por el bosque mesofítico, en el que abundan Laurus novocanariensis y Persea indica (L.) C.K. Spreng., entre otros. En las laderas de inclinación notable y en lomos de los interfluvios, el fayal-brezal con Myrica faya Aiton y Erica arbórea L., generalmente arbustivo, se hace dominante, para dar paso en las cotas superiores de la antigua Reserva (1100-1480 m s.m. aproximadamente), a un fayal-brezal-pinar de transición (pinar mixto) y por último al pinar genuino de Pinus canariensis C. Sm. ex DC. in Buch, propiamente dicho.

La superficie del paraje de El Canal y Los Tiles se distribuye en 14 cuadrículas de $1 \mathrm{~km}$ de lado (Fig. 1). A continuación se presenta una breve descripción de 16 localidades muestreadas en la zona de trabajo, con coordenadas UTM, altitud, exposición, pendiente, vegetación y especies de plantas dominantes, a las que se hace referencia mediante su correspondiente numeración al tratar cada uno de los táxones. Todas las localidades van precedidas de la misma información referida al país y división administrativa (ESPAÑA. Santa Cruz de Tenerife: La Palma, San Andrés y Sauces).

Loc. 1. Casa del Monte y alrededores: 28RBS2585, 1300-1400 m, NW, pendiente variable. Pinar mixto con Pinus canariensis, Erica arborea, Myrica faya, Dryopteris oligodonta (Desv.) Pic.-Serm (cuadrícula 11).

Loc. 2. Canal de Marcos y Cordero y alrededores: 28RBS2484, 1250 m, N-NW, 2 ${ }^{\circ}$. Pinar mixto con Cistus symphytifolius Lam., Cistus palmensis Bañares \& Demoly, Pinus canariensis, Erica arborea, Myrica faya (cuadrícula 13).

Loc. 3. Base del Espigón Atravesado: 28RBS2587, 550 m, N, $40^{\circ}$. Laurisilva de fondo de barranco con Ocotea foetens, Laurus novocanariensis, Hedera canarariensis Willd., Diplazium caudatum (cuadrícula 2).

Loc. 4. Fajana de los Revoltillos: 28RBS2586, $825 \mathrm{~m}, \mathrm{~N}-\mathrm{NE}, 15^{\circ}$. Laurisilva con Persea indica, Laurus novocanariensis, Picconia excelsa (Aiton) DC., Viburnum rigidum Vent., Hedera canariensis, Dryopteris oligodonta, Diplazium caudatum (cuadrícula 7).

Loc. 5. Puente Nuevo: 28RBS2586, 800 m, E-SE, 25․ Laurisilva con Laurus novocanariensis, Persea indica, Ilex canariensis Poir., Viburnum rigidum, Rubia peregrina L. subsp. agostinhoi (Dans. \& P. Silva) Valdés \& G. López, Dryopteris oligodonta (cuadrícula 7).

Loc. 6. Llano del Salto, parte alta de la Reserva: 28RBS 84,1425 $\mathrm{m}, \mathrm{W}-\mathrm{NW}, 30^{\circ}$. Pinar mixto con Pinus canariensis, Erica arborea, Myrica faya (cuadrícula 14).

Loc. 7. Fajana de Los Tiles: 28RBS2687, 550 m, N, 2 . Laurisilva con Ocotea foetens, Laurus novocanariensis, Hedera canariensis, Dryopteris oligodonta (cuadrícula 3).

Loc. 8. Bajada a Los Cortes: 28RBS2687, $500 \mathrm{~m}, \mathrm{~N}, 1-2^{\circ}$. Castaños plantados en laurisilva con Laurus novocanariensis, Myrica faya, Castanea sativa Mill., Rubus ulmifolius Schott (cuadrícula 3).

Loc. 9. Barranco del Agua o de Los Tiles, parte baja de la Reserva: $28 \mathrm{RBS} 2687,400-500 \mathrm{~m}, \mathrm{~N}, 1^{\circ}-25^{\circ}$. Laurisilva de ladera próxima al cauce de barranco con Ocotea foetens, Laurus novocanariensis, Diplazium caudatum (cuadrícula 3).

Loc. 10. Cuevas de Maldonado, sendero y alrededores: 28RBS2586, $1000 \mathrm{~m}, \mathrm{NW}, 2^{\circ}$. Fayal-brezal arbustivo de solana con Erica arborea, Myrica faya, Ilex canariensis, Dryopteris oligodonta, Asplenium onopteris L. (cuadrícula 7).

Loc. 11. Subida a Cuevas de Ventura: 28RBS2486, $850 \mathrm{~m}$, NW, $30^{\circ}$. Laurisilva con Laurus novocanariensis, Picconia excelsa, Dryopteris oligodonta (cuadrícula 6).

Loc. 12. Canal Chico: 28RBS2585, $1100 \mathrm{~m}, \mathrm{NE}, 30^{\circ}$. Laurisilva con Laurus novocanariensis, Ilex canariensis, Myrica faya, Dryopteris oligodonta (cuadrícula 11).

Loc. 13. Sendero del Espigón Atravesado a Cuevas del Espigón Grande: 28RBS2686, $700 \mathrm{~m}, \mathrm{NE}, 25^{\circ}$. Fayal-brezal arbustivo con Erica arborea, Ilex canariensis, Myrica faya, Laurus novocanariensis (cuadrícula 8). 


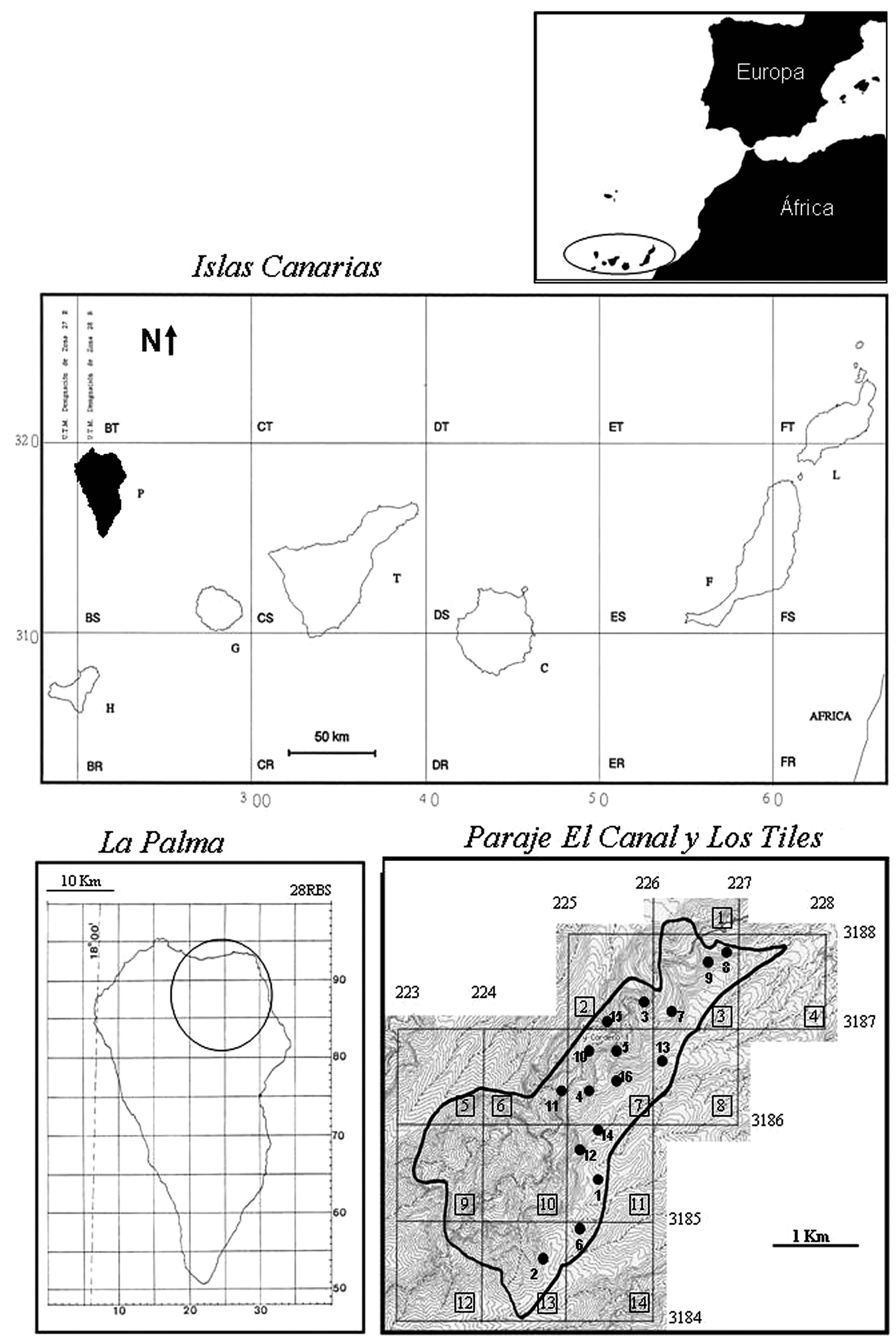

Fig. 1. Localización geográfica del área de estudio: paraje natural protegido de El Canal y Los Tiles (La Palma, Islas Canarias, España). 
Loc. 14. Fuente de Los Madroños: 28RBS2585, 875 m, NW, $30^{\circ}$. Laurisilva con Ocotea foetens, Laurus novocanariensis, Arbutus canariensis Veill., Rubus grex bollei Focke (cuadrícula 11).

Loc. 15. Topo de la Rabasa:28RBS2587, 1000 m, SE, 25․ Fayalbrezal con Erica arborea, Myrica faya, Viburnum rigidum, Ilex canariensis, Asplenium onopteris (cuadrícula 2).

Loc. 16. Fuente de Las Palomas: 28RBS2586, 800 m, NE, 1-2 Laurisilva con Ocotea foetens, Laurus novocanariensis, Genouinia arborea (L. f.) Gaudich, Salix canariensis C.Sm. ex Link. (cuadrícula 7).

\section{Material y métodos}

Se realizaron nueve campañas de tres días de duración cada una, a lo largo de tres años, desde diciembre de 1989 a diciembre de 1991. Se fijaron los momentos de visita en función de la climatología más favorable para el desarrollo de los hongos (otoño, invierno y primavera). Los muestreos se llevaron a cabo en 16 estaciones elegidas por su diversidad fúngica, dada la idoneidad de los hábitats observados in situ. La recolección de las muestras (455) siguió un patrón constante en la toma de datos abióticos y bióticos, reflejado en la descripción de las localidades y en la información contenida en cada taxón. Especial atención se puso a los sustratos en los que se desarrollaba el material objeto de estudio.

Las muestras fueron estudiadas a partir de material fresco y de herbario. En este último caso se rehidrataron en agua destilada y $\mathrm{KOH}$ al 5\%. Las muestras de Myxomycota fueron montadas en preparaciones permanentes utilizando el medio de Hoyer (Martin \& Alexopoulos, 1969). Los reactivos habituales fueron Melzer y Lugol (IKI) para evidenciar las propiedades amiloides y dextrinoides, Azul de Algodón para el carácter cianófilo y Rojo Congo para contrastar los elementos hialinos. Para los análisis macro y microscópico se utilizaron respectivamente un estereomicroscopio Zeiss mod. IV-b $(65 \times)$ y un microscopio óptico Olympus mod. BH-2. Para las micromedidas se utilizó un ocular micrométrico marca Wild $(15 \times$ SK). La iconografía que acompaña a la descripción de algunos táxones es original y ha sido realizada mediante una cámara clara de dibujo incorporada al microscopio óptico Olympus. Las muestras se hallan depositadas en el herbario TFC de la Universidad de La Laguna, sección de Micología (TFC Mic.). Para la nomenclatura de los Myxomycota se ha seguido a Lado (2001) y Lado \& al. (2005) y para el resto de los grupos se han consultado varias monografías y artículos en particular según los géneros tratados, además del Index Fungorum (indexfungorum.org/Names/Names.asp). Para la abreviatura de los nombres de los autores de táxones se han seguido las recomendaciones de Brummitt \& Powell (1992).

\section{Resultados}

\section{Táxones estudiados}

La relación de las especies estudiadas se presenta por orden alfabético dentro de cada grupo taxonómico: Myxomycota, Ascomycota y Basidiomycota. Con el fin de reducir el texto de este capítulo, la información del material examinado se ha abreviado en los siguientes términos: la categoría ecológica respecto al sustrato se indica mediante las siguientes abreviaturas: Te (terrícola), Fo (foliícola), Li (lignícola). En los dos últimos tipos se consigna a continuación la especie vegetal sustrato, si se ha identificado, y en caso contrario se indica solamente m.d. (s.i.) (madera en descomposición sin identificar). La localidad donde se ha recolectado la muestra se indica por su número. Los recolectores, salvo mención en el texto, son los autores del artículo. En el apartado de observaciones se consignan datos taxonómicos o si se trata de una novedad para La Palma o para Canarias, en su caso. La corología insular se abrevia mediante la letra inicial del nombre de las islas (H: Hierro; P: La Palma; G: Gomera; T: Tenerife; C: Gran Canaria; F: Fuerteventura; L: Lanzarote). Los datos de distribución en Canarias han sido tomados básicamente de Bañares (1988), Rodríguez-Armas \& Beltrán Tejera (1995), Beltrán Tejera (2004) y Beltrán Tejera \& al. (2004, 2008); y los de Madeira y Azores, de Dennis \& al. (1977) y Dennis (1986). El análisis de datos ecológicos y de biodiversidad sobre Agaricales y Aphyllophorales del capítulo final de este trabajo está basado en publicaciones anteriores propias, mencionadas en la introducción, y se ha actualizado la nomenclatura de algunas especies, siguiendo las fuentes bibliográficas ya indicadas.

\section{Myxomycota}

Arcyria affinis Rostaf., Sluzowce Monogr.: 276. 1875

Material estudiado: Loc. 9, 28-I-1989. Li, en m.d. (s.i.), (TFC Mic. 3399). Ibid., 2-XII-1989 (TFC Mic. 5123). Ibid., 14-IV1994 (TFC Mic. 5246).

Corología insular: Conocida de P, G, T.

Arcyria denudata (L.) Wettst., Verh. Zool.-Bot. Ges. Wien 35: Abh. 535. 1886

Material estudiado: Loc. 5, 12-II-1990. Li, m.d. (s.i.), J.M. Castro (TFC Mic. 5636).

Corología insular: Conocida de P, G, T.

Arcyria ferruginea Saut., Flora 24:316. 1841

Material estudiado: Loc. 9, 16-VII-1994. Li, en Ocotea foetens, J.M. Castro (TFC Mic. 5633).

Corología insular: Conocida de P, G, T.

Arcyria incarnata (Pers. ex J.F. Gmel.) Pers., Observ. Mycol. 1: 58. 1796

Material estudiado: Loc. 9, 16-III-1994. Li, en Ocotea foetens, J.M. Castro (TFC Mic. 9684).

Corología insular: Conocida de P, G, T, C. 
Arcyria pomiformis (Leers) Rostaf., Sluzowce Monogr.: 271. 1875 Material estudiado: Loc. 9, 16-III-1994. Li, en Erica arborea, J.M. Castro (TFC Mic. 9716).

Corología insular: Conocida de P, G, T, C.

Cribraria argillacea (Pers. ex J.F. Gmel.) Pers., Neues Mag. Bot. 1: 91. 1794

Material estudiado: Loc. 1, 2-XII-1989. Li, en m.d. (s.i.), (TFC Mic. 5565).

Corología insular: Conocida de P, G, T.

Cribraria cancellata (Batsch) Nann.-Bremek., Nederlandse Myxomyceten: 92.1975

Material estudiado: Loc. 9, 16-IX-1997. Li, en Ocotea foetens, J.M. Castro (TFC Mic. 8505, 9729). Corología insular: Conocida de P, G.

Hemitrichia abietina (Wigand) G. Lister in Lister, Monogr. Mycetozoa, ed. 2: 227. 1911

Material estudiado: Loc. 9, 14-IV-1994. Li, en Ocotea foetens, J.M. Castro (TFC Mic. 9698).

Corología insular: Conocida de P, G.

Hemitrichia calyculata (Speg.) M.L. Farr, Mycologia 66(5): 887. 1974

Material estudiado: Loc. 3, 10-II-1990. Li, en m.d. (s.i.), (TFC Mic. 5566).

Distribución insular: P.

Observaciones: Nuestro material muestra esporocarpos no densamente agregados, estipitados, con esporoteca subglosa a ovoide-turbinada, de hasta $1 \mathrm{~mm}$ de diámetro, de color amarillento ocráceo, que emerge abruptamente del ápice del pie. Peridio simple, delgado, membranáceo, de color amarillo naranja brillante, restando en la base como calículo, con bordes revolutos. Estípite cilíndrico, recto, de hasta $1,7 \mathrm{~mm}$ de altura, débilmente surcado, de color pardo oscuro intenso, amarillento al microscopio y relleno de cistes (10-15 $\mu \mathrm{m}$ de diámetro). Hipotalo membranáceo, de color marrón rojizo apagado. Capilicio tubular, con túbulos de 6-6,4 $\mu \mathrm{m}$ de diámetro, ramificados, con relieve de 4-5 bandas espiraladas, con escasos extremos libres. Esporas en masa de color amarillento, pálidas al microscopio, esféricas, de 7,5-9(11,7) um de diámetro, con ornamentación ténue, reticulada-espinulosa y con margen fino. Coincide con $H$. calyculata, a excepción de las medidas esporales que son mayores que las consignadas en la bibliografía (6-8 $\mu \mathrm{m}$ de diámetro, s.auct.). Esta especie es muy próxima a $H$. clavata (Pers.) Rostaf., de la cual es difícil separar, siendo considerada por algunos autores como sinónimos, mientras que otros dan argumentos más o menos consistentes para su tratamiento independiente. Lado \& Pando (1997) consideran como diferencias microscópicas más patentes las medidas esporales, mayores en $H$. clavata $(8-11 \mu \mathrm{m})$ y contorno esporal de hasta $1 \mu \mathrm{m}$ de grosor (apenas perceptible en $\mathrm{H}$. calyculata). Farr (1976) comenta ser incapaz de encontrar diferencias microscópicas significativas entre las dos especies. En nuestro caso y coincidiendo con Farr (1976) y Stephenson (2003), podría tratarse de una forma intermedia, como suele ocurrir entre táxones estrechamente relacionados. Nueva cita para Canarias. La muestra ha sido identificada por Carlos Lado.

Hemitrichia clavata (Pers.) Rostaf. in Fuckel, Jahrb. Nassauischen Vereins Naturk. 27-28: 75. 1873

Material estudiado: Loc. 9, 26-VI-1994. Li, en Ocotea foetens, J.M. Castro (TFC Mic. 3785).

Corología insular: G, T.

Observaciones: Las medidas de las esporas del material de La Palma (6,9-7,7 $\mu \mathrm{m}$ de diámetro; contorno esporal 1,2 $\mu \mathrm{m})$ son algo menores que las consignadas en la bibliografía (7-11 $\mu \mathrm{m}$ de diámetro, s.auct.). No obstante, el resto de caracteres micro y macroscópicos están de acuerdo con la bibliografía consultada
(Martin \& Alexopoulus, 1969; Farr, 1976; Nannega-Bremekamp, 1991; Lado \& Pando, 1997; Ing, 1999; Stephenson, 2003). Nueva cita para La Palma.

Leocarpus fragilis (Dicks.) Rostaf., Sluzowce Monogr.: 132. 1874 Material estudiado: Loc. 1, 30-IX-1991. Fo, facultativo, en acículas de Pinus canariensis (TFC Mic. 5145).

Corología insular: Conocida de P, G, T, C, y de Azores.

Lycogala epidendrum (L.) Fr., Syst. Mycol. 3: 80. 1829

Material estudiado: Loc. 5, 3-IV-1989. Li, en m.d. (s.i.), (TFC Mic. 3562). Loc. 1, 30-IV-1991. Li, en Pinus canariensis (TFC Mic. 3398). Loc. 9, 26-VI-1994. Li, en Ocotea foetens, J.M. Castro (TFC Mic. 3410).

Corología insular: Conocida de H, P, G, T, C, y de Madeira.

Stemonitis fusca Roth., Bot. Mag. (Römer \& Usteri) 1(2): 26. 1787 Material estudiado: Loc. 9, 8-IV-1994. Li, en Ocotea foetens, J.M. Castro (TFC Mic. 9685).

Corología insular: Conocida de P, G, T.

Stemonitis aff. splendens Rostaf., Sluzowce Monogr.: 195. 1874 Material estudiado: Loc. 9, 16-III-1994. Li, en m.d.(s.i.), J.M. Castro (TFC Mic. 9688).

Corología insular: Se conoce de T y de Madeira.

Observaciones: La identificación fue realizada por Carlos Lado, con reservas debido a la inmadurez del material estudiado. En caso de confirmarse se trataría de una nueva cita para La Palma.

Stemonitopsis typhina (F.H. Wigg.) Nann.-Bremek., Nederlandse Myxomyceten: 209. 1975 [“1974”]

Material estudiado: Loc. 5, 30-IV-1989. Li, en Ocotea foetens, J.M. Castro (TFC Mic. 3799). Loc. 9, 15-II-1994. Li, en Ocotea foetens (TFC Mic. 7079).

Corología insular: Se conoce de P, T.

Trichia botrytis (J.F. Gmel.) Pers., Neues Mag.Bot. 1: 89. 1794 Material estudiado: Loc. 3, 10-II-1990. Li, en m.d. (s.i.), J.M. Castro (TFC Mic. 5572). Loc. 9, 16-III-1994. Li, en Laurus novocanariensis (TFC Mic. 9719). Ibid., 14-IV-1994. Li, en Laurus novocanariensis y m.d. (s.i.), (TFC Mic. 9711, 9717). Loc. 16, 25-IV-1997. Li, en Ocotea foetens (TFC Mic. 8508, 9737).

Corología insular: Se conoce de P, G, T.

Trichia decipiens (Pers.) T. Macbr., N. Amer. Slime-Moulds: 218. 1899

Material estudiado: Loc. 16, 30-IV-1989. Li, en Salix canariensis (TFC Mic. 3780, 3781).

Corología insular: Se conoce de P, G, T.

Trichia favoginea (Batsch) Pers., Neues Mag. Bot. 1: 90.1794 Material estudiado: Loc. 3, 10.02.1990. Li, en m.d. (s.i.) (TFC Mic. 5564, 5567).

Corología insular: Se conoce de P, G.

Trichia persimilis P. Karst., Not. Sällsk. Fauna Fl. Fenn. Förh 9: 353. 1868

Material estudiado: Loc. 9, 24-IV-1994. Li, en Ocotea foetens, J.M. Castro (TFC Mic. 3910).

Corología insular: Se conoce de P, G, T.

Trichia subfusca Rex, Proc. Acad. Nat. Sci. Philadelphia 42: 192. 1890

Material estudiado: Loc. 16, 14-IV-1994. Li, sobre Persea indica, J.M. Castro (TFC Mic. 9678).

Corología insular: Se conoce de P, G, T.

Trichia varia (Pers. ex J.F. Gmel.) Pers., Neues Mag. Bot. 1: 90. 1794

Material estudiado: Loc. 9, 28-I-1989. Li, en m.d. (s.i.) y en Ocotea foetens (TFC Mic. 3400, 3409, 3401). Loc. 16, 30-IV-1989. Li, en Viburnum rigidum (TFC Mic. 3799). Loc. 4, 1-II-1991. Li, en m.d. (s.i.), J.M. Castro (TFC Mic. 7062).

Corología insular: Se conoce de P, G, T. 


\section{Ascomycota}

Aleuria aurantia (Pers.) Fuckel, Jb. Nassau. Ver. Naturk. 23-24: 325. 1870 [1869-70]

Material estudiado: Loc. 1, 12-XII-1987. Te (TFC Mic. 3605). Corología insular: Se conoce de P, T.

Annulohypoxylon michelianum (Ces. \& De Not.) Y.M.Ju, J.D. Rogers \& H.M. Hsieh, Mycologia 97(4): 859. 2005

Material estudiado: Loc. 16, 30-IV-1989. Li, en Erica arborea (TFC Mic. 3790). Loc. 9, 16-III-1994. Li, m.d. (s.i.), J.M. Castro (TFC Mic. 9666).

Corología insular: Se conoce de G, C.

Observaciones: Citada para Canarias como Hypoxylon michelianum Ces. \& De Not., por Urries (1957). Material identificado por J. Fournier y M. Stadler. Nueva cita para La Palma.

Annulohypoxylon minutellum (Syd. \& P. Syd.) Y.M. Ju, J.D. Rogers \& H.M. Hsieh, Mycologia 97(4): 859. 2005

Material estudiado: Loc. 8, 3-XII-1989. Li, en Castanea sativa (TFC Mic. 5170, 5172, 5191). Ibid., 16-III-1994, en Castanea sativa, J.M. Castro (TFC Mic. 9671, 9732).

Corología insular: Se conoce de P, G y de Madeira.

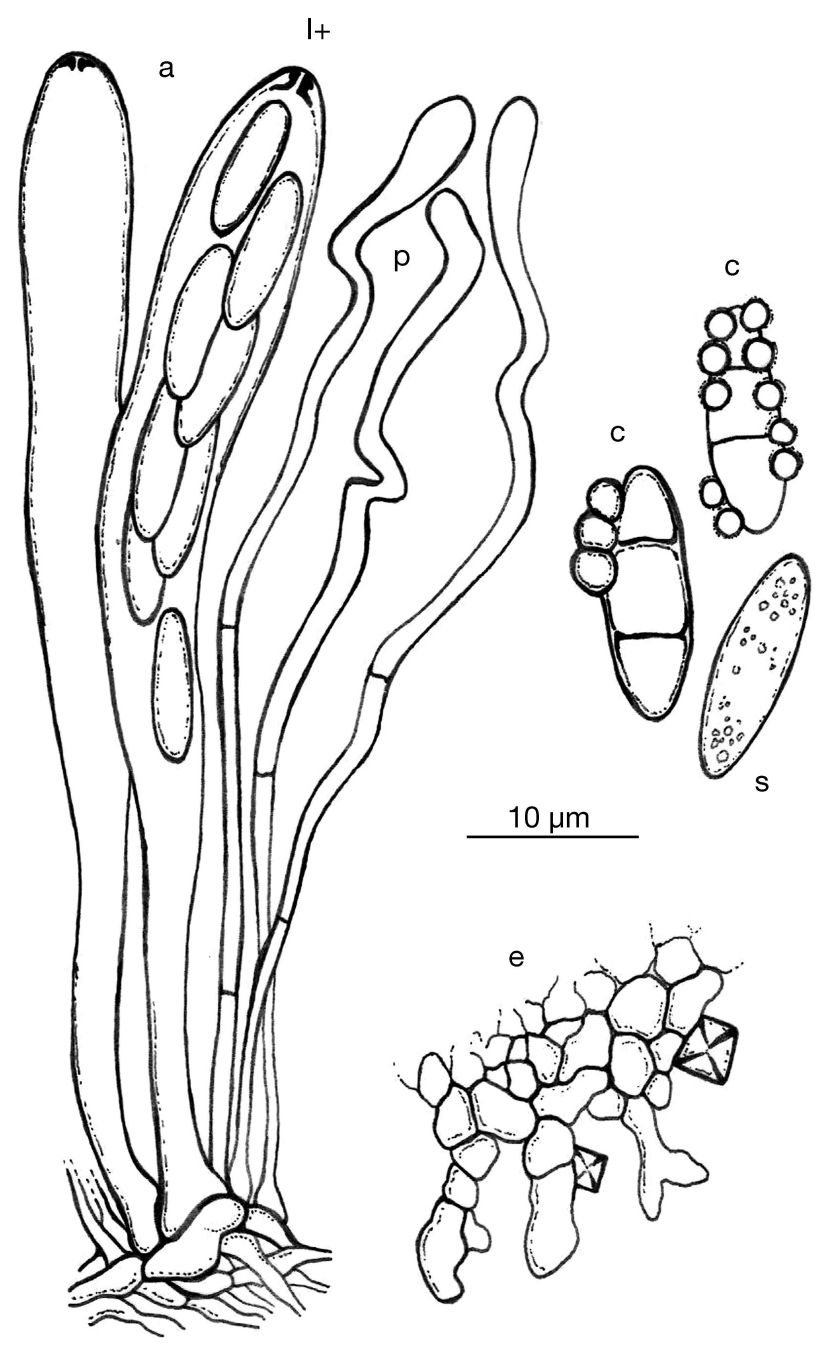

Fig. 2. Ascocoryne aff. cylichnium: a, ascas; p, parafisos; s, esporas; c, conidios; e, células del excípulo ectal (dibujos de L. Quijada).
Observaciones: Citada para La Palma como Hypoxylon coharens (Pers.: Fr.) Fr. (Dähncke, 1998; Karasch \& al., 2005). Para más información ver Beltrán Tejera \& al. (2008). El material ha sido identificado por J. Fournier y M. Stadler.

Ascocoryne aff. cylichnium (Tul.) Korf, Phytologia 21(4): 202. 1971

Material estudiado: Loc. 9, 28-I-1989. Li, en m.d. (s.i.) (TFC Mic. 3413, duplic. in CUP-MM-2740).

Corología insular: P.

Observaciones: El material canario muestra apotecios ciatiformes, sésiles o subestipitados, gelatinosos, de 2-4 mm de diámetro, de color negro-parduzco con tintes púrpuras. Excípulo ectal formado por células redondeadas a vermiformes, irregularmente curvadas o con proyecciones laterales, hinchadas, con incrustaciones de cristales de oxalato. Excípulo medular de textura intricada, gelatinizada, células de 3,8-4,5 $\mu \mathrm{m}$ de ancho. Ascas 130-137 × 6-7 $\mu \mathrm{m}$ en material seco de herbario, cilíndrico-clavadas, poro amiloide, azul en IKI, partiendo de croziers. Parafisos filiformes, septados, hinchados en el ápice $(3,5 \mu \mathrm{m})$. Esporas elíptico-fusoides, de 16-18 × 4,8-5,1 $\mu \mathrm{m}$, aparentemente multigotuladas en vivo, finalmente 1-3 septadas; conidios esféricos, independientes, emergiendo directamente de las ascósporas y unidos a ellas, de 2,1-2,6 × 1,9-2,5 $\mu \mathrm{m}$ (Fig. 2). El material estudiado coincide en parte de sus caracteres morfológicos con los contenidos en las descripciones bibliográficas de $A$. cylichnium (Dennis, 1978; Roll-Hansen \& Roll-Hansen, 1979; Breitenbach \& Kränzlin, 1984; Hansen \& Knudsen, 2000; Baral \& Marson, 2005), excepto por el menor tamaño de los apotecios $(6-30 \mathrm{~mm}$, s.auct. $)$ y de las esporas $(18-33 \times 4-6 \mu \mathrm{m}$, s.auct.); número de septos de las mismas (multiseptadas, s.auct.); y ancho de las células excipulares de la médula (8-12 $\mu \mathrm{m}, s$. Baral in litt.). Estos caracteres diferenciales coinciden más con Ascocoryne sarcoides (Jacq. ex S.F. Gray) Groves \& Wilson (próxima a $A$. cylichnium) y a la cual se podría asimilar el material estudiado, si no fuera por que $A$. sarcoides no posee incrustaciones de oxalato en las células del excípulo ectal y por sus ascoconidios unidos en cadenas. El excipulum medular observado en $\mathrm{KOH}$ es gelatinizado, si bien no se ha podido determinar si es alta o débilmente gelatinizado, ya que se trata de material seco de herbario. Después de remitir al Dr. Baral toda la información taxonómica y gráfica y siguiendo sus consejos, hemos creído oportuno mantener este taxon como afín a A. cylichnium a la espera de material vivo adicional. De confirmarse la identificación de esta especie, se trataría de una nueva cita para Canarias. Por otra parte, Ascocoryne sarcoide fue citada para Tenerife (Beltrán Tejera \& al., 1989), a partir de material confirmado por el Dr. Korf (TFC Mic. 3177, duplic. in CUP-MM2619).

Bisporella claroflava (Grev.) Lizoñ \& Korf, Mycotaxon 54: 474. 1995

Material estudiado: Loc. 16, 30-IV-1989. Li, en Laurus novocanariensis (TFC Mic. 3787). Loc. 9, 23-III-1994. Li, sobre m.d. (s.i.) y Laurus novocanariensis, J.M. Castro (TFC Mic. 9673, 9776). Ibid., 19-IX-1997. Li, en Castanea sativa (TFC Mic. 5702).

Corología insular y mundial: Se conoce de P, G, T.

Observaciones: Las características morfológicas de los especímenes estudiados coinciden con la descripción de Dennis (1978) para Bisporella sulfurina (Quél.) S.E. Carp., nombre de la nueva combinación que propuso Carpenter en Korf \& Carpenter (1974), para Helotium sulfurinum Quél. No obstante, en un trabajo posterior Korf \& Zhuang (1985), expresaron sus dudas sobre si la común Bisporella sulfurina puede ser realmente separada de Bisporella discedens (P. Karst.) S.E. Carp. Sobre este último taxon Lizoñ \& Korf (1995) hicieron un detallado comentario histórico de su nomenclatura y justificaron su coes- 
pecifidad con Helotium citrinicolor P. \& H. Crouan y Peziza claroflava Grev., basándose en el estudio de los especímenes tipos respectivos. Estos autores concluyeron que Peziza claroflava es el nombre prioritario, la incluyeron en el género Bisporella y establecieron la nueva combinación (Bisporella claroflava).

Ante la posible coespecifidad de B.sulfurina y B.claraflava y siguiendo los consejos del Dr. Baral (in litt.), hemos adoptado el concepto de Korf \& Zhuang (1985). Karasch \& al. (2005) citaron Bisporella claroflava para La Palma (San Isidro, Breña Alta), creciendo sobre Chamaecytisus proliferus (L.f.) Link subsp. proliferus var. palmensis (Christ) Hansen \& Sunding.

Chlorociboria aeruginascens (Nyl.) Kanouse ex C.S. Ramamurthi, Korf \& L.R. Batra, Mycologia 49(6): 858. 1958 [1957] Material estudiado: Loc. 5, 12-XII-1987. Li, en m.d. (s.i.), (TFC Mic. 3607)

Loc. 7, 28-I-1989. Li, en m.d. (s.i.), (TFC Mic. 3437). Ibid., 01IV-1989. Li, en Ocotea foetens (TFC Mic. 3540).

Corología insular: Se conoce de P, G, T.

Coccomyces delta (Kunze) Sacc., Bol. Soc. Broteriana, Coimbra 11: 13.1893

Material estudiado: Loc. 9, 20-II-1994. Fo, en hojas de Ocotea foetens (TFC Mic. 9692).

Corología insular y mundial p.p.: P, G, C. Se ha citado para la Península Ibérica (Vizcaya), sobre hojas de Laurus nobilis (Undagoitia \& al., 2007).

Observaciones: El material estudiado mostraba ascocarpos deltoides-piramidales, ocasionalmente cuadrangulares, menores de $1 \mathrm{~mm}$, de color negro, inmersos en los tejidos epidérmicos del huésped, rodeados de anillos necróticos de color negro. Ascas cilíndricas, poro no amiloide, de 98-120 × 9,4-10 $\mu \mathrm{m}$. Parafisos cilíndricos, septados, no ramificados, débilmente ensanchados en el ápice, de 150-165 × 3-4,5 $\mu \mathrm{m}$. Esporas filiformes, hialinas, multiseptadas, de 73-77 × 1,5-2 $\mu \mathrm{m}$. Urries (1957) citó esta especie por primera vez para Canarias (La Palma: Cubo de La Galga y Bco. del Agua o Los Tiles; La Gomera; y Gran Canaria), sobre hojas caídas de distintas lauráceas (Laurus novocanariensis, Ocotea foetens y Persea indica). La muestra de Los Tiles estudiada por nosotros coincide en sus caracteres de diagnóstico con la descripción de Coccomyces delta (Dennis, 1978; Undagoitia \& al. 2007). Johnston (1986) describió de Nueva Zelanda una especie muy próxima, Coccomyces lauraceus P.R. Johnst. y comenta la coincidencia de caracteres con el material de Coccomyces delta, recolectado por Korf \& al., en Azores, Madeira y Canarias (La Palma, Los Tilos, sobre Ocotea sp.). Se diferencia básicamente por poseer perífisis que se organizan en una capa excipular y por sus esporas significativamente más cortas y más anchas $(41-60 \times 2,3-3 \mu \mathrm{m})$. Coccomyces lauraceus ha sido citada con posterioridad para Australia (Johnston, 2000).

Daldinia macaronesica M. Stadler, Wollw. \& J.M. Castro, Mycol. Res. 108(3): 259. 2004

Material estudiado: Loc. 7, 28-II-1989. Li, en Ocotea foetens (TFC Mic. 3421, 3428). Ibid., 2-XII-1989. Li, en Ocotea foetens (TFC Mic. 5201).

Corología insular y mundial: Se conoce de P, G, T, C.

Observaciones: Esta especie ha sido citada para Canarias erróneamente por diferentes autores como Daldinia concentrica (Bolton: Fr.) Ces. \& De Not. Un reciente estudio de Stadler \& al. (2004), basado en material de La Palma (Cubo de La Galga y Los Tiles) y Madeira (Fanal), en el que se llevan a cabo, entre otros, análisis quimiotaxonómicos (HPLC) y caracteres del anamorfo, dieron como resultado la descripción de una nueva especie, $D$. macaronesica, que es independiente de $D$. concentrica. Dähncke (1998) mencionó esta especie como D. concentrica para Los Tiles. Para una información más detallada sobre este taxón, distribución y sustratos ver Beltrán Tejera \& al. (2008).
Una segunda especie, Daldinia palmensis M. Stadler, Wollweber \& H-.V.Trichy, descrita (Stadler \& al., 2004) de material canario (La Palma, La Galga), no ha sido hallada aún en el paraje de El Canal y Los Tiles. Las muestras estudiadas han sido identificadas por J. Fournier y M. Stadler.

Discinella aff. boudieri (Quélet) Boud. Hist. Classific. Discom. Europe: 96. 1907

Material estudiado: Loc. 4, 29-XI-1991. Te, creciendo entre briófitos (TFC Mic. 6384).

Corologia insular: P.

Observaciones: Nuestro material mostraba apotecios ciatiformes, de 2-5,8 mm diámetro, umbilicados, lisos, de color pardo anaranjados (rehidratados) y carne gelatinosa. Excípulo ectal de textura intricada. Excípulo medular de textura porrecta. Ascas cilíndrico-clavadas, con un lado del poro apical amiloide, débilmente azul en IKI, de 108-111 × 9-10 $\mu \mathrm{m}$, partiendo de croziers. Parafisos cilíndricos a sublanceolados, septados, rami-

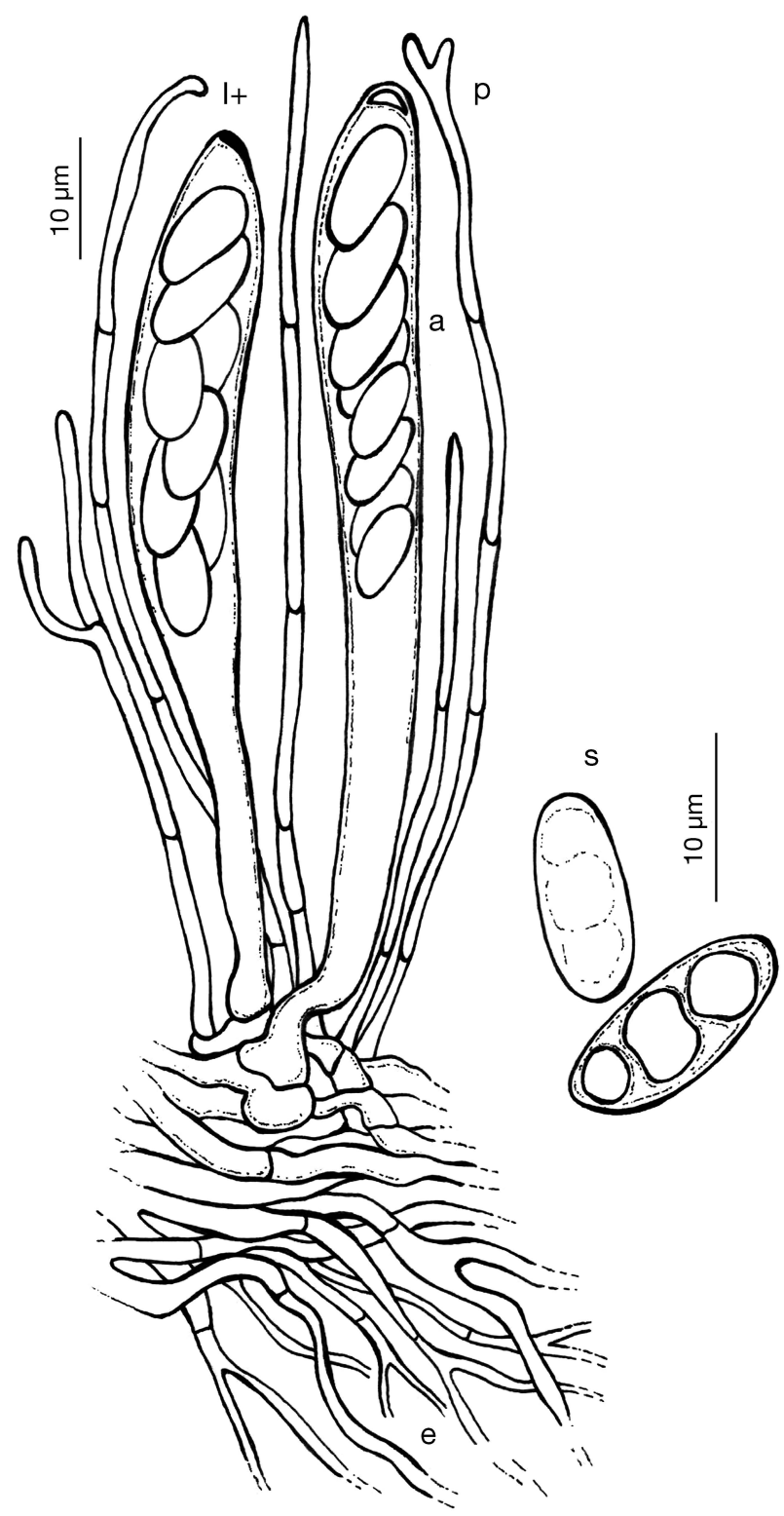

Fig. 3. Discinella aff. boudieri: a, ascas; $\mathbf{p}$, parafisos; s, esporas; e, células del excípulo medular (dibujos de L. Quijada). 
ficados, de 89-105 × 1,2-2,5 $\mu \mathrm{m}$. Esporas de 14-15 × 6,5-7,8 $\mu \mathrm{m}$, hialinas, con 3 grandes gótulas en su interior en $\mathrm{KOH}$ en la madurez (Fig. 3).

Las características morfológicas del material estudiado coinciden con Discinella boudieri, excepto la medida del ancho de las esporas, mayor que el indicado en la bibliografía (6,5-7,8 vs. 45 m) (Dennis, 1978; Baral \& Marson, 2005; Hansen \& Knudsen, 2000). En opinión del Dr. Baral, a quien hemos remitido toda la información taxonómica y gráfica de la muestra, el material de La Palma está muy próximo a D.boudieri, pero las medidas del ancho de las esporas aconsejan realizar estudios futuros y más detallados en material vivo. De confirmarse su identificación se trataría de una nueva cita para Canarias.

Geoglossum umbratile Sacc., Michelia 1(no. 4): 444.1878 Material estudiado: Loc. 2, 2-IV-1989. Te (TFC Mic. 3609). Corología insular: Se conoce de T, C.

Observaciones: Nueva cita para La Palma.

Helvella lacunosa Afzel.: Fr., Kongl. Vetensk. Acad. Nya Handl. 4:303. 1783

Material estudiado: Loc. 12, 11-II-1990. Te (TFC Mic. 5609). Corología insular: Se conoce de H, P, T, C.

Hypocrea rufa (Pers.) Fr., Summa veg. Scand., Section Post. (Stockholm): 383. 1849

Material estudiado: Loc. 9, 12-XII-1987. Li, en m.d. (s.i.) (TFC

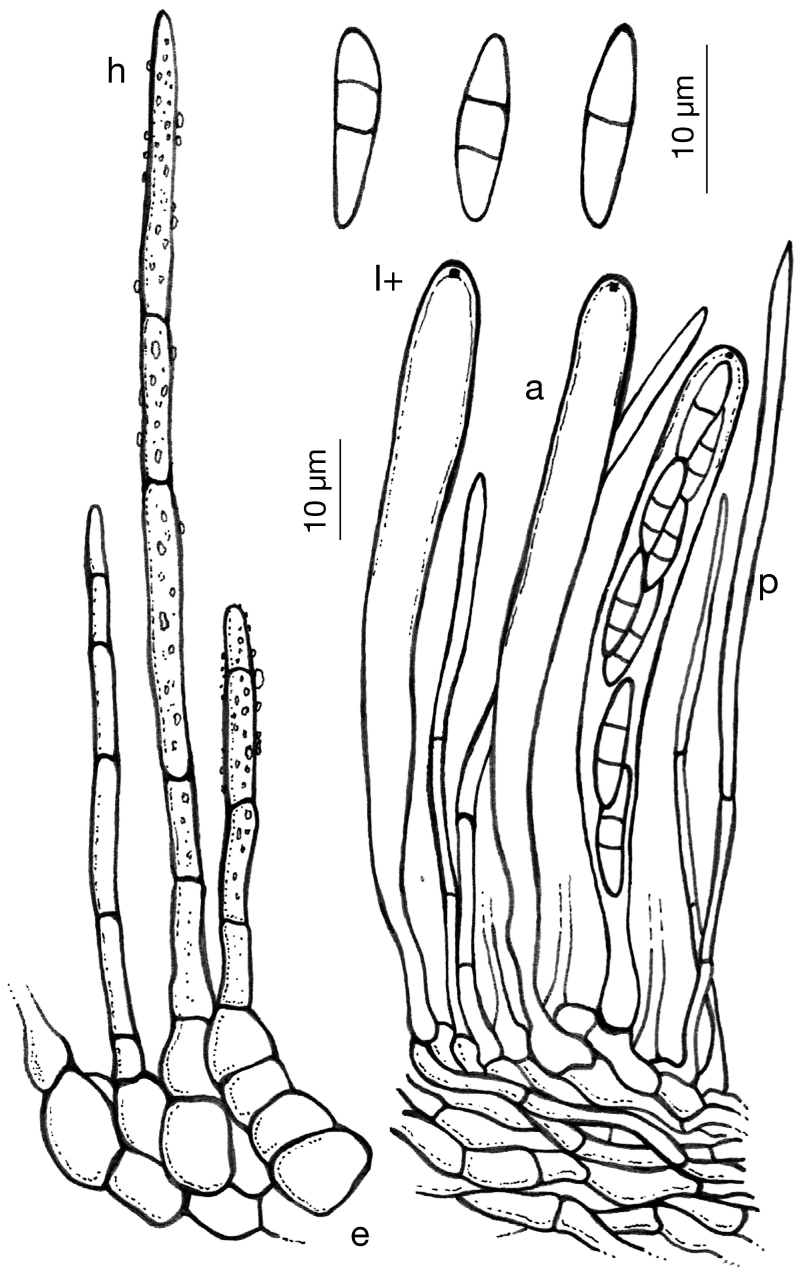

Fig. 4. Lasiobelonium sulflavidum: a, ascas; p, parafisos; s, esporas; e, células del excípulo ectal; $\mathbf{h}$, pelos (dibujos de L. Quijada).
Mic. 5648). Ibid. 28-I-1989. Li, en m.d. (s.i.) (TFC Mic. 3418). Ibid., 30-IV-1989. Li, en Persea indica (TFC Mic. 3788).

Corología insular: Se conoce de P, G, H.

Lachnum virgineum (Batsch) P. Karst., Bidr. Känn. Finl. Nat. Folk 19: 169. 1871

Material estudiado: Loc. 14, 28-III-1989. Li, en Rubus gr. bollei (TFC Mic. 3436).

Corología insular: Se conoce de P, G, T y de Azores.

Lasiobelonium subflavidum Ellis \& Everh., Bull. Torrey bot. Club 24: 136. 1897

[= Dasyscyphus triseptatus Dennis, Kew Bulletin 15:300. 1961] Material estudiado: Loc. 9, 23-III-1984. Li, en Laurus novocanariensis (TFC Mic. 9721).

Corología insular: $\mathrm{P}, \mathrm{T}$.

Observaciones: El material estudiado mostraba ascocarpos ciatiformes, subsésiles, de 0,27-0,7 mm de diámetro, de color pardoblanquecino, cubiertos externamente de pelos blancos. Excípulo ectal de textura globuloso-angular, células de 4,6-10,2 × 3,5-7,3 $\mu \mathrm{m}$. Excípulo medular de textura porrecta, hifas discurriendo paralelamente en la misma dirección. Pelos cilíndricos, parduscos en la base y hialinos en el ápice, septados, recubiertos de gránulos, de 53-117 × 2,3-3,4 $\mu \mathrm{m}$. Ascas cilíndrico-clavadas, con poro amiloide, azul en IKI, de 59-98 × 4,8-7,6 $\mu \mathrm{m}$. Parafisos cilíndricos-sublanceolados de 64-119 × 1,2-3 $\mu \mathrm{m}$, tabicados y simples. Esporas fusoides, con abundantes gotas lipídicas, de 1-3 septos, de 8,9-15,9 × 1,8-3,3 $\mu \mathrm{m}$ (Fig. 4). Estas características coinciden con las aportadas por Raitviir (1980), Spooner (1987) y Baral \& Marson (2005), si bien las medidas del ancho del asca y largo de esporas entran en los límites inferiores de dichas descripciones y la longitud de los pelos es inferior a la indicada por estos autores. No obstante, el Dr. Baral, una vez vistas la información taxonómica y la gráfica enviadas para su estudio, coincide en la adscripción de este material a L. subflavidum. Además, él había ya recolectado una muestra de esta especie en Tenerife, Monte de las Mercedes, sobre ramas sin identificar, el 27-II-1978 (H.B. 2243) (in litt.). Nueva cita para Canarias.

Mollisia sp. (1)

Material estudiado: Loc. 13, 10-II-1990. Li, sobre Erica arborea (TFC Mic. 5578). Loc. 3, 2-XII-1989. Li, en el mismo sustrato (TFC Mic. 5212).

Observaciones: Las muestras presentaban ascocarpos discoides o pateliformes, sésiles, con margen lobulado, de 4-5 mm de diámetro, de color marrón grisáceo, con el borde blanco y disco amarillo-ocráceo (rehidratado), decolorándose a amarillo en $\mathrm{KOH}$; base exterior del apotecio con hifas hialinas, a modo de anclajes. Ascas cilíndricas, poro amiloide, azul en IKI, de 76$100 \times 6,5-8,8 \mu \mathrm{m}$. Parafisos cilíndricos, simples o ramificados, de 75-120 $\mu \mathrm{m}$ de largo, ápice ligeramente ensanchado $(\times 2,8$ $3,4 \mu \mathrm{m})$. Esporas fusiformes, subcurvadas, hialinas, $0-1$ septadas y con pequeñas gótulas, de 13-17 × 2,3-3 $\mu \mathrm{m}$. Estas características morfológicas coinciden en gran parte con las descripciones bibliográficas de Mollisia ramealis $\mathrm{P}$. Karst. encontradas en la bibliografía (Dennis, 1978; Breitenbach \& Kränzlin, 1984), a excepción del tamaño de los apotecios y ancho de las ascas (hasta $1 \mathrm{~mm}$ de diámetro y $\times 8 \mu \mathrm{m}$, respectivamente, en $M$. ramealis). Esta especie no ha sido citada para Canarias.

Mollisia sp. (2)

Material estudiado: Loc. 9, 12-XII-1987. Li, en Ocotea foetens (TFC Mic. 3603, duplic. in CUP-MM-2742).

Observaciones: El material estudiado puede corresponder a Mollisia ventosa $\mathrm{P}$. Karst. ya que coincide en la mayoría de sus caracteres, pero difiere por poseer ascósporas biseriadas (uniseriadas en $M$. ventosa) y parafisos ensanchados en el ápice $(\times 2,4$ $3 \mu \mathrm{m})$ (menores o iguales a $1 \mu \mathrm{m}$ en $M$. ventosa). Esta especie ha sido citada con anterioridad para La Palma (Dähncke, 1998), sin indicar localidad. 
Dada la dificultad taxonómica de este género, su notable representación en los bosques de laurisilva canaria y por haber estudiado sólo material seco de herbario, hemos optado por mantener nuestra identificación a nivel genérico.

Nectria peziza (Tode: Fr.) Fr., Summa Veg. Scand., Section Post. (Stockholm): 388. 1849

Material estudiado: Loc. 7, 29-XI-1991. Li, en Ocotea foetens (TFC Mic. 18630).

Corología insular: Se conoce de G y de Azores

Observaciones: Nueva cita para La Palma.

Nemania serpens (Pers.) Gray, Nat. Arr. Brit. Pl. (London) 1:508, 516. 1821

Material estudiado: Loc. 9, 28-I-1989. Li, en m.d. (s.i.) (TFC Mic. 3426)

Corología insular: Se conoce de P.

Observaciones: Nuestra muestra ha sido confirmada por J. Fournier y M. Stadler. Citada para La Palma por Dähncke (1998), sin indicar localidad ni ecología.

Otidea cochleata (Huds.) Fuckel, Jb. Nassau. Ver. Naturk. 23-24: 329. 1870 [1869-70]

Material estudiado: Loc. 1, 2-XII-1989. Te (TFC Mic. 5118).

Corología insular: Se conoce de P y de Azores.

Observaciones: Citada para La Palma por Dähncke (1998), sin indicar localidad ni ecología.

Propolis farinosa (Pers.) Fr., Summa veg. Scand., Section Post. (Stockholm): 372.1849

[= Propolis versicolor $(\mathrm{Fr}.) \mathrm{Fr}$.

Material estudiado: Loc. 1, 28-I-1989. Li, en Myrica faya (TFC Mic. 3572).

Corología insular: Se conoce de P, G, T y de Azores.

Rhizodiscina lignyota (Fr.) Hafellner, Beih. Nova Hedwigia 62: 195. 1979

Material estudiado: Loc. 9, 12-XII-1987. Li, en m.d. (s.i.) (TFC Mic. 3583).

Corología insular: P, G.

Observaciones: La colección estudiada mostraba apotecios dispersos, discoide-pateliformes, de 0,5-1 mm de diámetro, sésiles, lisos, de color negro, margen crenulado, disco gelatinoso (rehidratado), de color ocre negruzco. Ascas claviformes, de paredes gruesas en el ápice (bitunicadas), de base subestipitada, amiloides, octosporadas, de (24)-56 × (8)-12 $\mu \mathrm{m}$. Parafisos cilíndricos, septados-moniliformes, con ápice ensanchado-capitado $(\times 3-4 \mu \mathrm{m})$ embebido en un gel. Esporas biseriadas, obovoides o clavadas, bicelulares (con una célula más ancha que la otra), de color pardo, lisas, de (7,2)-11-(15) × (3,6)4-5 $\mu \mathrm{m}$. Esta especie la hemos recolectado también en el Parque Nacional de Garajonay (La Gomera), en Myrica faya (30-XI-2002, TFC Mic. 14317) y Chamaecytisus proliferus (29-XI-2001, TFC Mic. 14388). Nuestro material no presenta el himenio de color amarillo-anarajando que comenta Sierra López (2006), probablemente se trate de un carácter visible en estado joven y fresco. Nueva cita para Canarias.

Sarcoscypha macaronesica Baral \& Korf, in Baral, Z. Mykol. 50(1): 124.1984

Material estudiado: Loc. 5, 12-XII-1987. Li, en m.d. (s.i.) (TFC Mic. 3604). Ibid., 2-IV-1989. Li, en Laurus novocanariensis (TFC Mic. 3557). Ibid., 1-II-1991. Li, en m.d. (s.i.) (TFC Mic. 7061). Loc. 9, 28-II-1989. Li, en m.d. (s.i.) (TFC Mic. 3425). Loc. 13, 10-II-1990. Li, en m.d. (s.i.) (TFC Mic. 5586).

Corología insular: Se conoce de P, G, T, C y de Madeira. Especie exclusiva de la Región Macaronésica (Baral \& Korf, 1984, in Korf \& Zhuang, 1991).

Observaciones: Ha sido citada por Dähncke (1998), para Los Tiles.

Scutellinia scutellata (L.) Lambotte, Mém. Soc. Roy. Sci. Liège, Série 2, 1:299. 1887
Material estudiado: Loc. 2, 1-IV-1989. Te, en la pared de un canal de agua, en tierra y musgos (TFC Mic. 3465 y 3462). Loc. 5, 1-IV-1990. Te (TFC Mic. 3568).

Corología insular: Se conoce de P, G, T, también de Madeira y Azores.

Observaciones: Esta especie ha sido citada por Dähncke (1998), para Los Tiles.

Stictis radiata (L.) Pers., Observ. Mycol. (Lipsiae) 2: 73. 1800 [1799]

Material estudiado: Loc. 15, 1-IV-1989. Li, en Myrica faya (TFC Mic. 3526). Loc. 5, 12-II-1990. Li, en Rubus gr. bollei (TFC Mic. 5643). Loc. 12, 11-II-1990. Li, en Myrica faya (TFC Mic. 5828). Loc. 9, 28-III-1994. Li, en m.d. (s.i.), J.M. Castro (TFC Mic. 9713). Corología insular: Se conoce de P, G, T.

Stictis stellata Wallr., Fl. crypt. Germ. (Nürnberg) 2: 444. 1833 Material estudiado: Loc. 5, 28-II-1989. Li, en m.d. (s.i.), C. Hernández (TFC Mic. 7058).

Corología insular: Se conoce de P.

Tarzetta catinus (Holmk.) Korf \& J.P. Rogers, Phytologia 21(4): 206. 1971

Material estudiado: Loc. 1, 03-IV-1989. Te (TFC Mic. 3452). Corología insular: Se conoce de P, T, C.

Trichoglossum hirsutum (Pers.: Fr.) Boud., Hist. Class. Discom. Eur. (Paris): 86. 1907

Material estudiado: Loc. 4, 28-I-1989. Te (TFC Mic. 3416). Loc. 9, 18-I-1994. Te, J.M. Castro (TFC Mic. 6633).

Corología insular: Se conoce de P, G, T y de Madeira.

Xylaria hypoxylon (L. ex Hooker) Grev., Fl. Edin.: 355. 1824 Material estudiado: Loc. 4, 23-III-1996. Li, en m.d. (s.i.), enterrada (TFC Mic. 5569).

Corología insular: Se conoce de P, G, T, C y de Madeira.

Xylaria polymorpha (Pers. ex Mérat) Grev., Fl. Edin.: 355. 1824 Material estudiado: Loc. 9, 1-II-1991. Li, en Ocotea foetens, J.M. Castro (TFC Mic. 10265).

Corología insular: Se conoce de P, G, T.

\section{Basidiomycota}

Auricularia auricula-judae (Bull.) Quél., Enchir. Fung. (Paris): 207. 1886

Material estudiado: Loc. 7, 1-IV-1989. Li, en Ocotea foetens (TFC Mic. 3543). Loc. 9, 11-XII-1989. Li, en el mismo sustrato (TFC Mic. 3595).

Corología insular: Se conoce de P, G, T, C.

Observaciones: Ha sido citada por Wildpret \& al. (1973) y Dähncke (1998), para Los Tiles.

Bovista aestivalis (Bonord.) Demoulin, Beih. Sydowia 8: 143. 1979 Material estudiado: Loc. 1, 2-XII-1989. Te (TFC Mic. 5161). Corología insular: Se conoce de H, P, G, T, C, F.

Calocera cornea (Batsch) Fr., Stirp. Agri. Femison. 5: 67. 1827 Material estudiado: Loc. 1, 11-II-1990. Li, en m.d. (s.i.) (TFC Mic. 5607).

Corología insular: Se conoce de P, G.

Observaciones: Nueva cita para La Palma.

Dacrymyces capitatus Schwein., Trans. Am. phil. Soc., Ser. 2, 4(2): 186. 1832

Material estudiado: Loc. 1, 1-IV-1989. Li, en Cistus aff. symphytifolius (TFC Mic. 3467). Loc. 16, 30-IV-1989. Li, en Gesnouinia arborea (TFC Mic. 3786).

Corología insular: Se conoce de P, G, T, y de Azores.

Dacrymyces variisporus MacNabb, N.Z. Jl Bot. 11(3): 504. 1973 Material estudiado: Loc. 1,30-XI-1991. Li, en Pinus canariensis (TFC Mic. 3407).

Corología insular: Se conoce de P, G, T. 
Geastrum fimbriatum Fr., Syst. Mycol. (Lundae) 3(1): 16. 1829 Material estudiado: Loc. 1, 3-IV-1989. Te (TFC Mic. 3454). Loc. 5, 1-II-1991. Te (TFC Mic. 5579). Loc. 2, 2-II-1991. Te (TFC Mic. 9712).

Corología insular: Se conoce de P, G, T.

Geastrum triplex Jungh., Tijdschr. Nat. Gesch. Physiol. 7: 287. 1840

Material estudiado: Loc. 9, 28-I-1989. Te (TFC Mic. 3432). Loc. 1, 2-XII-1989. Te (TFC Mic. 5163). Loc. 5, 2-XII-1989. Te (TFC Mic. 5211, 5532, 5233). Loc. 14, 2-XII-1989. Te (TFC Mic. 5236). Loc. 12, 11-II-1990. Te (TFC Mic. 5626).

Corología insular: Se conoce de P, G, T.

Laurobasidium lauri (Geyl.) Jülich, Int. J. Mycol. Lichenol. 1(1): 118. 1982

Material estudiado: Loc. 13, 10-II-1990. Tumoraciones vegetales producidas por el hongo microscópico, en el tronco vivo de Laurus novocanariensis (TFC Mic. 5587). Loc. 9, 30-I-1991, en el mismo sustrato ( $\sin$ recol.)

Corología insular y mundial: Se conoce de P, G, T, C. En Canarias y Madeira parasita a Laurus novocanariensis, en Azores a L. azorica y en Galicia, Portugal, Italia y Marruecos a L. nobilis (Beltrán Tejera \& al., 2008).

Observaciones: Esta especie ha sido citada por Beltrán Tejera \& Wildpret de la Torre (1975), para Los Tiles.

Lycoperdon lambinonii Demoulin, Lejeunia, n.s. 62: 13. 1972 Material estudiado: Loc. 5, 1-II-1991. Te (TFC Mic. 3762). Corología insular: Se conoce de H, P, G, T, C.

Phallus impudicus L., Sp. pl.: 1178. 1753

Material estudiado: Loc. 5, 2-XII-1989. Te (TFC Mic. 5222). Loc. 8, 3-XII-1989. Te (TFC Mic. 5165).

Corología insular: Se conoce de H, P, G, T, C y de Madeira.

Rhizopogon roseolus (Corda) Th. Fr., Svensk Bot. Tidskr. 1(3): 282. 1909

Material estudiado: Loc. 1, 2-XII-1989. Te (TFC Mic. 5112, 5252)

Corología insular: Se conoce de H, P, G, T, C y de Madeira.

Scleroderma bovista Fr., Syst. mycol. (Lundae) 3(1): 48.1829 Material estudiado: Loc. 9, 28-II-1989. Te (TFC Mic. 3433). Loc. 1, 2-XII-1989. Te (TFC Mic. 5148)

Corología insular: Se conoce de P, T.

Scleroderma polyrhizum (J.F. Gmel.) Pers., Syn. Meth. Fung. (Göttingen) 1: 156.1801

Material estudiado: Loc. 9, 29-II-1989. Te (TFC Mic. 3438). Corología insular: Se conoce de P, G, T, C.

Sebacina calcea (Pers.) Bres., Fung. Trident. 2: 64. 1898

[= Exidiopsis calcea (Pers.) K. Wells $]$

Material estudiado: Loc. 3, 28-II-1989. Li, en Ilex canariensis (TFC Mic. 3429). Loc. 10, 1-IV-1989. Li, en raíz aflorada de Myrica faya (TFC Mic. 3585). Loc. 8, 3-XII-1989. Li, en Rubus ulmifolius (TFC Mic. 5180)

Corología insular: G, T.

Observaciones: Nueva cita para La Palma.

Tremella foliacea Pers., Observ. Mycol. (Lipsiae) 2: 98.1800 [1799] Material estudiado: Loc. 8, 3-XII-1989. Li, en Salix canariensis (TFC Mic. 5184).

Corología insular: Se conoce de P, G.

Tremella mesenterica Retz., K. Svenska Vetensk-Akad. Handl. 30: 249. 1769

Material estudiado: Loc. 1, 1-IV-1989. Li, en Myrica faya (TFC Mic. 3458). Loc. 1, 2-XII-1989. Li, en raíz aflorada de Myrica faya (TFC Mic. 5249). Loc. 9, 30-I-1990. Li, en Laurus novocanariensis (TFC Mic. 3536). Loc. 12, 11-II-1990. Li, en tronco vivo de Erica arborea (TFC Mic. 5617). Loc. 6, 11-II-1990. Li, en m.d. (s.i.) (TFC Mic. 5603). Loc. 1, 2-XII-1990. Li, en Myrica faya (TFC Mic. 5138).

Corología insular: Se conoce de P, G, T, C y de Madeira.

\section{Discusión y conclusiones}

En este trabajo se presenta un total de 66 especies: 21 Myxomycota, 29 Ascomycota y 16 Basidiomycota, de las que 11 se citan por primera vez para La Palma. De estas novedades, Hemitrich a calyculata (Myxomycota), Lasiobelonium subflavidum y Rhizodiscina lignyota (Ascomycota) se mencionan por primera vez para Canarias; además, 2 especies de Ascomycota (Ascocoryne aff. cylichnium y Discinella aff. boudieri), cuya adscripción específica definitiva queda pendiente, pero que en cualquier caso se trata de táxones no citados hasta el momento para el Archipiélago. Por otra parte, excepto seis especies (Coccomyces delta, Daldinia macaronesica, Sarcoscypha macaronesica, Scutellinia scutellata, Auricularia auricula-judae y Laurobasidium lauri), los táxones estudiados se citan por primera vez para el paraje de El Canal y Los Tiles.

Las 21 especies de Myxomycota estudiadas (7,6\% del total) se distribuyen en 8 géneros, entre los que destacan Trichia, con 6 especies, seguido de Arcyria (5) y Hemitrichia (3). El orden Trichiales supone, pues, más del 66\% de las especies de este grupo. Este patrón parece repetirse aproximadamente en otros bosques de laurisilva de Canarias (Beltrán Tejera \& al., 2008). Con respecto a los grupos de Basidiomycota relacionados en esta comunicación, a pesar igualmente del bajo número de especies registradas (16; $5,8 \%)$, resultan también habituales en este tipo de ambientes (op.cit.).

Ascomycota se haya representado por 32 especies (11,6\% de la micobiota total), incluidas 3 de Hypoxylon, que publicamos con anterioridad (Stadler \& al., 2008): Hypoxylon crocopeplum Berk. \& M.A. Curtis, Hypoxylon subrutilum Starbäck e Hypoxylon canariense, esta última descrita como novedad para la ciencia a partir de material de Tenerife, Gran Canaria, La Gomera y de varias localidades de la isla de La Palma, particularmente bien representada en El Canal y Los Tiles. Somos conscientes de que este número es notablemente inferior a lo esperado en los bosques de laurisilva canarios para el grupo (Beltrán Tejera \& al., 2008). Probablemente se deba en parte a la recolección no sistemática que se llevó a cabo en su momento, que dio como resultado un mayor volumen de muestras de especímenes pertenecientes a grupos con cuerpos fructíferos más evidentes (Agaricales y Aphyllophorales), y que fueron objeto de una mayor atención por nuestra parte. Sabemos por experiencia que órdenes como Helotiales y Xylariales entre otros, se hallan muy bien representados en estos ecosistemas boscosos y que necesitan ser exhaustivamente estudiados. Un dato curioso de Ascomycota es su riqueza genérica, ya que las 32 especies estudiadas se incluyen 
en 26 géneros. Sólo Annulobypoxylon, Mollisia, Stictis y Xylaria están representados por dos especies cada uno, e Hypoxylon por tres.

Sin lugar a dudas, el orden con mayor diversidad específica en esta localidad es Agaricales, del que hemos dado a conocer en publicaciones anteriores un total de 112 especies, lo que representa el 40,6\% de la micobiota total de la zona (Bañares \& al., 1992, 1994; Esteve-Raventós \& al., 1998; Bañares \& Arnolds, 2002). Entre los géneros con mayor representación específica destacan Mycena (16 especies), Hygrocybe (11), Inocybe (8), Tricholoma (7), Clitocybe (5), Cortinarius (3) y Pluteus (3). Le sigue en importancia el grupo de Aphyllophrales s.l., del que hemos publicado hasta el momento 95 especies, lo que representa el 34,4 \% del total (Rodríguez-Armas \& al., 1992a,b, 1994; Larsen \& al., 1994; Rodríguez-Armas \& Beltrán Tejera, 1995), con un alto porcentaje de corticiáceos s.l. $(44,2 \%)$, incluidos en distintos géneros y entre los que podemos mencionar Hyphoderma, Peniophora, Sistotrema y Scytinostroma, con 3 especies cada uno. Otros géneros, pertenecientes a otras familias y que merecen mención son Ramaria y Stereum (5 especies, respectivamente), e Hymenochaete, Phellinus y Trametes, con 3 especies cada uno.

Si sumamos todos los datos numéricos expuestos, hemos dado a conocer un total de 276 táxones fúngicos para El Canal y Los Tiles (Fig. 5). A esta cifra se han de añadir las citas de Dähncke (1998), para esta localidad, no mencionadas en este trabajo y que se refieren a cuatro especies de Agaricales: Cystolepiota pulverulenta (Huijsman) Vellinga, Flammulaster limulatus (Fr. ex Weinm.) Watling, y dos especies más, al parecer nuevas para la ciencia: "Leucoagaricus lateritius Mohr et Dähncke ad int." y "Leucocoprinus astriatus Mohr et Dähncke ad int.", pero para las que no da diagnosis latina ni asigna los tipos nomenclaturales respectivos. Con posterioridad, Mohr enmendó la primera identificación de "Leucoagaricus lateritius" (nom. nudum), asimilando el material a Leucoagarius purpureolilacinus Huijsman (Dähncke, in litt.). Por otra parte, "Leucocoprinus astriatus" (nom. nudum), fue válida y efectivamente publicada como una nueva especie para la ciencia, con el nombre de Leucoagaricus rosemarieae P. Mohr (Mohr, 2007). Igualmente se ha de añadir para este paraje la cita de Arenal \& al. (2008) de Cotylidia muscigena L. Relmy.

Como hemos mencionado anteriomente, la laurisilva es la vegetación dominante de este paraje y, en consecuencia, el mayor número de estaciones muestreadas corresponde a este tipo de formación (10 de 16). En fayal-brezal y en pinar mixto se han muestreado 3, respectivamente.

La Tabla 1 recoge los resultados cuantitativos globales obtenidos de los muestreos realizados y que incluyen todos los grupos fúngicos estudiados por nosotros (incluidos en este trabajo y los anteriores publicados): De las 276 especies estudiadas en total, 201 $(72,8 \%)$ se han registrado en las diferentes variantes de laurisilva (comunidades de fondo de barranco y de laderas de mayor o menor pendiente, con presencia relativamente frecuente de Persea indica y otros elementos arbóreos, o más próximas al cauce con mayor abundancia de Ocotea foetens). De estas especies, 122 ( $44,2 \%$ del total) han sido recolectadas exclusivamente en esta formación y entre ellas se encuentran la mayor parte de Myxomycota (18 especies de 21; 85,7\%) y de Ascomycota (22 de 32; 68,7\%); casi la mitad de especies catalogadas de Aphyllophorales (43 de 95; $45,2 \%$ ) y algo menos de la mitad de especies de Agaricales ( 43 de 112;38,4\%). Para estos dos últimos grupos, no tratados en este trabajo, podemos mencionar: Clavulinopsis fusiformis (Sowerby) Corner, Clavulinopsis helvola (Pers.) Corner, Junghubnia nitida (Fr.)

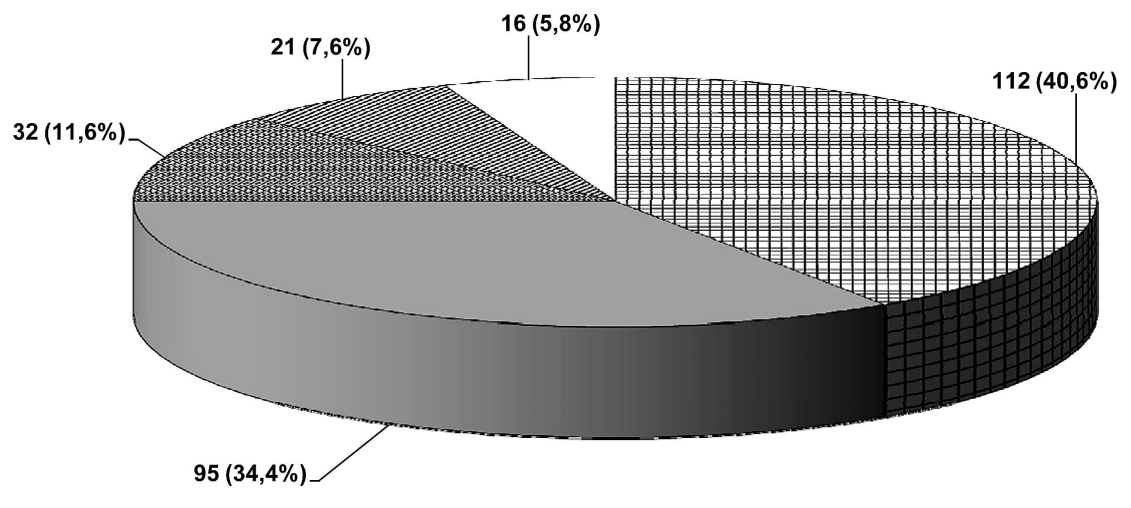

घAgaricales 口Aphyllophorales $⿴$ Ascomycota $\square$ Myxomycota $\square$ O.Basid.

Fig. 5. Número de especies y porcentajes respectivos, pertenecientes a los diferentes grupos fúngicos estudiados. 
Tabla 1. Número de especies por grupo fúngico y su distribución en los distintos tipos de comunidades vegetales presentes en el área de estudio.

\begin{tabular}{|c|c|c|c|}
\hline & Laurisilva & Fayal-brezal & Pinar mixto \\
\hline Aphyllophorales .. & 59 & 21 & 36 \\
\hline Agaricales ............. & 86 & 2 & 77 \\
\hline O. Basidiomycota . & 11 & 2 & 11 \\
\hline 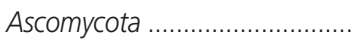 & 27 & 3 & 7 \\
\hline 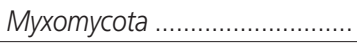 & 18 & - & 3 \\
\hline Total (especies acumuladas) & 201 & 28 & 134 \\
\hline
\end{tabular}

Ryvarden, Mycoacia uda (Fr.) Donk, Pblebia livida (Pers.) Bres., Scytinostroma ochroleucum (Bres. \& Torrend) Donk, Rigidoporus ulmarius (Sowerby) Imazeki, Trechispora farinacea (Pers.) Liberta, Trametes ochracea (Pers.) Gilb. \& Ryvarden, Hemimycena mauretanica (Maire) Singer, Lentinellus cocbleatus (Pers.) P. Karst., Mycena acicula (Schaeff.) P. Kumm., M. alba (Bres.) Kühner, Mycenella margaritispora (J.E. Lange) Singer, Pleuroflammula raggaziana (Bres.) Horak, $\mathrm{Hy}$ grocybe reai (Maire) J.E. Lange, H. russocoriacea (Berk. \& Jos. K. Mill.) P.D. Orton \& Watling, H.psittacina (Schaeff.) P. Kumm., Pleurotus dryinus (Pers.) P. Kumm., etc. Menor es la presencia exclusiva en laurisilva de otros Basidiomycota ( 5 de 16;31,2\%), como es el caso de Auricularia auricula-judae, Tremella foliacea y los gasteromicétidos Lycoperdon lambinonii, Phallus impudicus y Scleroderma polyrbizum. Estas tres últimas especies han sido observadas también en comunidades de pinar mixto en otras localidades del archipiélago (Beltrán Tejera \& al. 2004).

En las tres estaciones de fayal-brezal muestreadas se recolectaron sólo 28 especies $(10,1 \%)$, la mayor parte de las cuales pertenece al orden Aphyllophorales $(21 ; 22,1 \%)$. Sólo 2 de Agaricales [Crepidotus mollis (Schaeff.) Staude, también observada en laurisilva, y Crepidotus cesatii (Rabenh.) Sacc., esta última registrada únicamente en fayal-brezal]. Igualmente 2 especies de otros Basidiomycota (Laurobasidium lauri y Sebacina calcea, ambas también de laurisilva y esta última recolectada además en pinar mixto). Ascomycota se halla representada en esta unidad ambiental por 3 especies, que también se repiten en laurisilva (Mollisia aff. ramealis, Sarcoscypha macaronesica y Stictis radiata). No se recolectó ninguna especie de Myxomycota en fayal-brezal.

Por último, en las tres estaciones situadas en cotas superiores, correspondientes al pinar mixto (pinar con fayal-brezal), se contabilizaron 134 especies, de las que $85(30,8 \%)$ se observaron únicamente en esta formación, entre las que se pueden mencionar: $\mathrm{Cri}$ braria argillacea, Leocarpus fragilis, Aleuria aurantia, Geoglossum umbratile, Otidea cochleata, Propolis farinosa, Tarzetta catinus, Calocera cornea, Dacrymyces variisporus, Bovista aestivalis y Rhizopogon roseolus. Agaricales es el grupo mejor representado en el pinar mixto, con 77 especies $(68,7 \%)$, de las cuales aproximadamente la mitad $(55 ; 49,1 \%)$ se observaron exclusivamente en esta unidad ambiental. Entre las más frecuentes se encuentran: Agaricus campestris L., Amanita gemmata (Fr.) Bertill., Cortinarius elatior Fr., Hebeloma crustuliniforme (Bull.) Quélet, Hygrophoropsis aurantiaca (Wulfen) Maire, Suillus bellinii (Inzenga) Kuntze, Tricholoma focale (Fr.) Ricken, T. saponaceum (Fr.) P. Kumm., T. terreum (Schaeff.) P. Kumm., etc.; y entre las raras: Clitocybe anisata Velen., Cortinarius acutus (Pers.) Fr., Inocybe pudica Kühner, Melanoleuca robertiana Bon, etc. Finalmente, Aphyllophorales

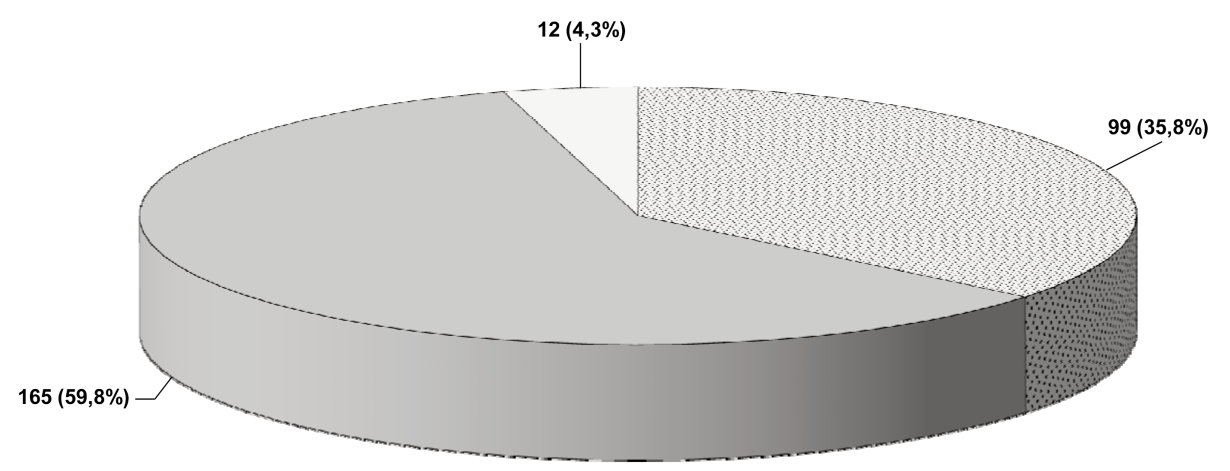

⿴囗大 Terrícolas $\square$ Lignícolas $\square$ Foliícolas

Fig. 6. Número de especies y porcentajes, de los diferentes grupos fúngicos, atendiendo a las distintas categorías ecológicas respecto a los sustratos. 
se halla representado en el pinar mixto por 36 especies $(37,9 \%)$, de las que $21(22,1 \%)$ se recolectaron exclusivamente en esta formación forestal. Entre estas últimas podemos mencionar: Aleurodiscus aurantius (Pers.) J. Schröt., Heterobasidium annosum (Fr.) Bref., Phellodon niger (Fr.) P. Karst., Sistotrema confluens Pers., S. pteriphilum Larss. \& Hjortstam, Stereum gausapatum (Fr.) Fr., Thelephora anthocephala Fr., T. terrestris Fr., Tomentella oligofibula, T. terrestris (Berk. \& Br.) M.J. Larsen, etc.

El resto de las especies en su conjunto registrada en pinar mixto, también se observaron en otra formación vegetal, la mayoría en laurisilva, exhibiendo por tanto una cierta amplitud ecológica. Entre éstas podemos mencionar: Lycogala epidendrum, Chlorociboria aeruginascens, Scutellinia scutellata, Dacrymyces capitatus, Geastrum fimbriatum, Geastrum triplex, Scleroderma bovista, Tremella mesenterica, Clitocybe decembris Singer, C.rivulosa (Pers.) P. Kumm., Mycena galericulata (Scop.) Gray, Hypholoma fascicularis (Huds.) P. Kumm., Ramaria flaccida (Fr.) Ricken, Pblebiopsis ravenelii (Cooke) Hjortstam, Terana caerulea (Lam.) Kuntze, Radulomyces confluens (Fr.) P. Christ., etc. Es de destacar que las especies de mayor distribución son 5 Aphyllophorales: Peniophora lycii (Pers.) Höhn. \& Litsch., Steccherinum ochraceum (Pers.) Gray, Phellinus torulosus (Pers.) Bourdot \& Galzin, Schizopora radula (Pers.) Hallenb. y Stereum reflexulum D.A. Reid, registradas en los tres tipos de formaciones forestales presentes en el área de estudio.

En cuanto a la categoría ecológica respecto a los sustratos, dominan las lignícolas (Fig. 6), con un total de
165 especies $(59,8 \%)$, distribuidas de la siguiente manera: 20 especies (95,2\%) de Myxomycota; 20 especies (62,5\%) de Ascomycota; 39 especies (34,8\%) de Agaricales, entre los que se incluyen 2 especies carbonícolas [Faerberia carbonaria (Alb. \& Schwein.) Pouzar y Pholiota highlandensis (Peck) A.H. Sm. \& Hesler]. La mayor proporción de lignícolas corresponde a Aphyllophorales, con 75 especies (78,9\%). Finalmente, en el resto de Basidiomycota tratados en este trabajo se hallan 8 especies lignícolas (50\%), correspondientes a los heterobasidiomicétidos, además de Laurobasidium lauri (ustilaginomicétido), parásito microscópico de Laurus novocanariensis. La fidelidad de los hongos saprótrofos a una determinada especie vascular es rara, por lo que es relevante la presencia constante de Auricularia auricula-judae en los ecosistemas de laurisilva canarios, ligada a Ocotea foetens. Sólo 12 especies $(4,3 \%)$ se observaron sobre hojas caídas (foliícolas): Leocarpus fragilis, Coccomyces delta, Athelopsis lembospora (Bourdot) Oberw., Botryobasidium obtusisporum J. Erikss., Litschauerella clematitis (Bourdot \& Galzin) J. Erikss. \& Ryvarden, Parvobasidium cretatum (Bourdot \& Galzin) Jülich, Flammulaster carpophilus (Fr.) Earle, Marasmius budsonii (Pers.) Fr., Marasmius epiphylloides var. latispora, Mycena adonis (Bull.) Gray, Mycena urania (Fr.) Chalet y Mycena rorida (Scop.) Quélet.

Finalmente, los terrícolas suman 99 especies (35,8\%), la mayoría pertenecientes a Agaricales (67 especies; $59,8 \%)$. El resto se distribuyen en: 16 especies (16,8\%) de Aphyllophorales; 8 especies (50\%) de otros Basidiomycota, correspondientes a los gastero-

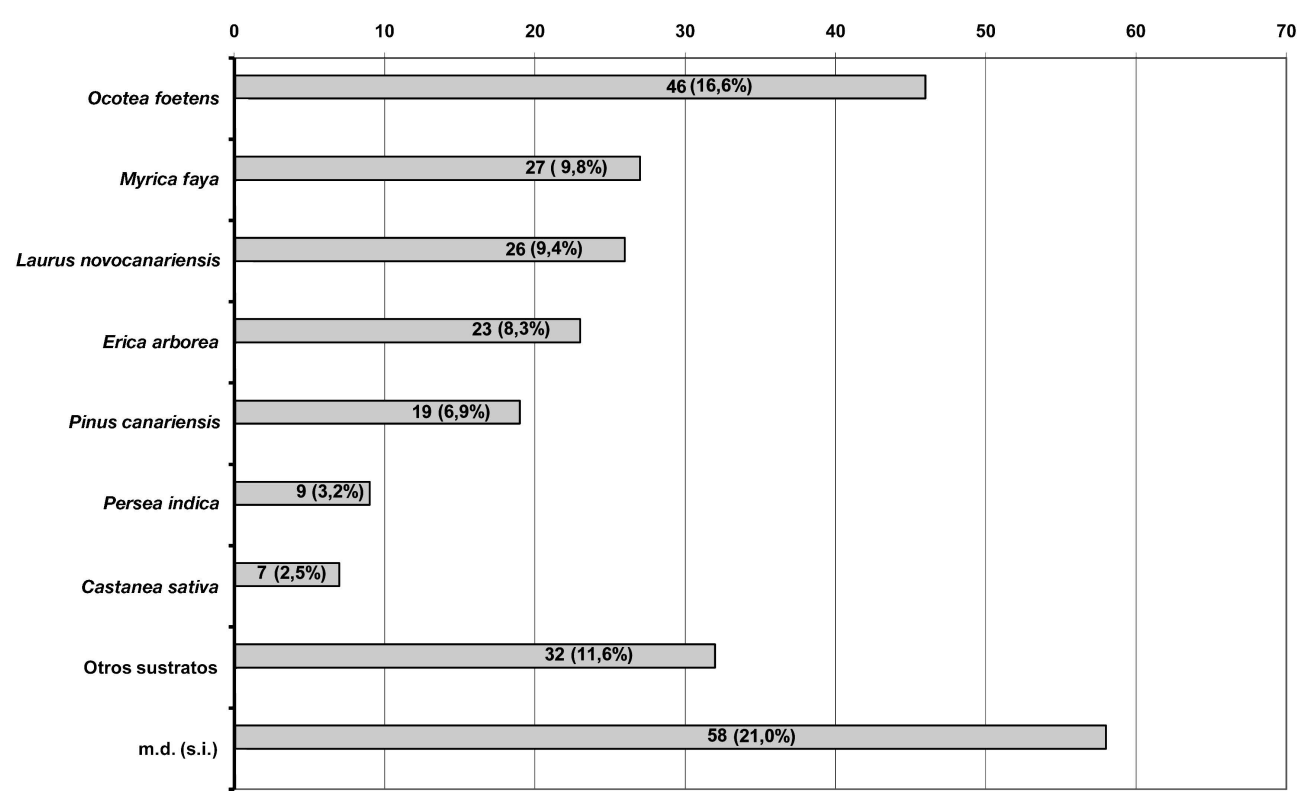

Fig. 7. Número de especies y porcentajes, recolectadas en las diferentes especies de plantas vasculares sustratos. 
micétidos; igualmente 8 (25\%), de Ascomycota. Entre los Myxomycota no se registró ninguna especie terrícola.

La mayor proporción de lignícolas es una constante en las formaciones de laurisilva canarias, categoría ecológica dominante en los Aphyllophorales ya comentados. Mientras que entre los Agaricales dominan los terrícolas (Beltrán Tejera \& al., 1989, 2008; Rodríguez-Armas \& Beltrán Tejera, 1995).

Se identificaron 22 especies de plantas vasculares en cuyos restos leñosos, hojas caídas o incluso árboles vivos, se han observado los hongos y mixomicetes catalogados. El sustrato vascular más importante resultó ser Ocotea foetens, en el cual se registraron 46 especies (16,6\%), una de ellas en hojas caídas (Coccomyces delta). A notable distancia le sigue Myrica faya, con 27 especies $(9,8 \%)$, una en hojas caídas [Hemimycena crispula (Quélet) Singer]; en Laurus novocanariensis se registraron 26 especies $(9,4 \%)$, una de ellas en árboles vivos (Laurobasidium lauri) y otra en hojas caídas [Mycena rorida (Scop.) Quélet]; en Erica arborea 23 especies (8,3\%); en Pinus canariensis 19 especies (6,9\%), una de ellas en acículas (Leocarpus fragilis); en Persea indica 9 especies (3,2\%); y en Castanea sativa 7 especies $(2,5 \%)$. Un total de 32 especies fúngicas $(11,6 \%)$ se registraron en otras especies vasculares, en número inferior a 5 por sustrato, por lo que hemos globalizado el dato en una sola columna (Fig. 7).

Un número relativamente alto de especies (58; $21 \%$ ), fueron recolectadas sobre madera en un estado tan avanzado de descomposición que fue imposible su identificación. No obstante, este porcentaje no es significativo dada la mezcla de especies vasculares que representa (Fig. 7).

\section{Agradecimientos}

Queremos expresar nuestro sincero agradecimiento a H.O. Baral, Carlos Lado, Richard P. Korf, Marc Stadler y Jacques Fournier, por la identificación o confirmación de algunos táxones. A Juan Manuel Castro, por la recolección de varias muestras que se dan a conocer en este trabajo. A la Unidad Insular de Medio Ambiente de La Palma, por haber puesto a nuestra disposición el Centro que se encuentra en Los Tiles, en el cual desarrollamos parte de la investigación de campo. El proyecto fue subvencionado por la Dirección General de Investigación Científica y Técnica del Ministerio de Educación y Ciencia (DGICYT, ULL-N ${ }^{\circ}$ PB871004).

\section{Referencias bibliográficas}

Arenal, F., Villarreal, M. \& Rubio, V. 2008. Cotylidia muscigena, a new record from Canary Islands. Cryptogamie, Mycologie 29 (1): 19-23.

Bañares, Á. (1988). Hongos de los Pinares de Tamadaba. Instituto de Estudios Canarios (C.E.C.E.L.)-Consejo Superior de Investigaciones Científicas. Monografía XXXVI. Tenerife. 280 pp.
Bañares, Á. \& Arnolds, E. 2002. Hygrocybe monteverdae. A new species of subgenus Cuphophyllus (Agaricales) from the Canary Islands (Spain). Persoonia 18(1): 135-138.

Bañares, A., Beltrán Tejera, E. \& Rodríguez-Armas, J.L.1992. Estudio micológico de la Reserva de la Biosfera El Canal y Los Tiles (La Palma, Islas Canarias). II. Agaricomycetidae ( $1^{a}$ Parte). Documents Mycologiques 22(86) 47-64.

Bañares, A., Beltrán E. \& Rodríguez-Armas, J.L. 1994. Estudio micológico de la Reserva de la Biosfera El Canal y Los Tiles (La Palma, Islas Canarias). III. Agaricomycetidae (2 $2^{a}$ Parte). Cryptogamie, mycologie 15(1): 1-20.

Baral, H.-O. \& Marson, G. 2005. In vivo veritas. Over 10000 Images of Fungi and Plants (microscopical drawings, water colour plates, photo macro- \& microphraphs), with materials on vital taxonomy and xerotolerance. DVD, 3nd edition.

Beltrán Tejera, E., 2004. Fungi. In: Izquierdo, I., Martín, J.L., Zurita, N. \& Arechavaleta, M. (eds.), Lista de especies silvestres de Canarias (hongos, plantas y animales terrestres) 2004. Consejería de Medio Ambiente y Ordenación Territorial, Gobierno de Canarias. p: $21-57$.

Beltrán Tejera, E. \& Wildpret de la Torre, W. 1975. Táxones nuevos en la flora fúngica canaria. Vieraea 5(1-2): 127-166.

Beltrán Tejera, E., Bañares Baudet, Á., Rodríguez-Armas, J.L., Losada Lima, A. \& León Arencibia, M.C. 1989. Contribución al estudio de la flora micológica del Monte de Aguas y Pasos (Los Silos, Tenerife). III. Documents mycologiques 19(76): 41-58.

Beltrán Tejera, E., Rodríguez-Armas, J.L., Bañares Baudet, A., Barrera Acosta, J. \& Lado Rodríguez, C. 2004. Hongos. In: Beltrán Tejera, E. (Ed.), Hongos, Líquenes y Briófitos del Parque Nacional de La Caldera de Taburiente. O.A. de Parques Nacionales, Serie Técnica. Ministerio de Medio Ambiente. Madrid: 55-232.

Beltrán Tejera, E., Rodríguez-Armas, J.L., Bañares, A. \& Lado, C. 2008. Hongos. In: Beltrán Tejera, E. (ed.), Hongos, Liquenes y Briófitos del Parque Nacional de Garajonay (La Gomera, Islas Canarias). O.A. de Parques Nacionales, Serie Técnica. Ministerio de Medio Ambiente. Madrid: 41-389.

Breitenbach, J. \& Kränzlin, F. 1984. Champignons de Suisse. Tome 1. Les Ascomycetes. Société Mycol. Lucerne. Ed. Mykologia, CH 6000 Lucerne. 310 pp.

Brummitt, R.K. \& Powell, C.E. 1992. Authors of Plant Names. Publ. Royal Botanical Gardens, Kew. 732 pp.

Dähncke, R.M.1998. Las Setas en La Palma. Excmo. Cabildo Insular de La Palma y Caja General de Ahorros de Canarias. 413 pp.

Dennis, R.W.G. 1970. Fungus flora of Venezuela and adjacent countries. Kew Bull. Add. Series III. Royal Botanic Gardens, Kew. 531 pp.

Dennis, R.W.G. 1978. British Ascomycetes. J. Cramer, Vaduz, XVI $+585 \mathrm{pp}$.

Dennis, R.W.G. 1986. Fungi of the Hebrides. Royal Bot. Gard. Kew, Whitstable Litho Ltd., Whitstable, Kent, 383 pp.

Dennis, R.W.G., Reid, D.A. \& Spooner, B. 1977. The Fungi of the Azores. Kew Bulletin 32: 85-136.

Esteve-Raventós, F., Bañares, Á., Beltrán Tejera, E. \& Rodríguez, J.L. 1998. Estudio micológico de la Reserva de la Biosfera El Canal y Los Tiles (La Palma, Islas Canarias). IV. Agaricomycetidae ( $3^{a}$ Parte). Género Inocybe. Cryptogamie, mycologie 19(12): 121-129.

Farr, M.L. 1976. Myxomycetes. Flora Neotropica 16: 1-305.

Hansen, L. \& Knudsen, H. (eds.) 1997. Nordic Macromycetes. Vol. 3 Heterobasidioid, Aphyllophoroid and Gastromycetoid, Basidiomycetes. Helsinki University Printing House, 444 pp.

Hansen, L. \& Knudsen, H. (eds.) 2000. Nordic Macromycetes. Vol. 1 Ascomycetes. Helsinki University Printing House, 309 pp. 
Ing, B. 1999. The Myxomycetes of Britain and Ireland: An Identification Handbook. The Richmond Publishing Co. Ltd. England. $374 \mathrm{pp}$

Jhonston, P.R. 1986. Rhytismataceae in New Zealand 1. Some foliicolous species of Coccomyces de Notaris and Propolis (Fries) Corda. New Zealand Journal of Botany 24: 89-124.

Jhonston, P.R. 2000. Rhytismatales of Australia: the Genus Coccomyces. Australian Systematic Botany 13: 199-243

Karasch, P., Dämon, W., Jaklitsch, W. \& Baral, H.O. 2005. Beiträge zur Pilzflora der Kaneren-Insen La Palma 2. Weitere bemerkenswerte Pilzfunde auf Chamaecytisus proliferus. Österr. Z. Pilzk. 14:275-289.

Korf, R.P. \& Carpenter, S.E. 1974. Bisporella, a generic name for Helotium citrinum and its allies, and the generic names Calycella and Calycina. Mycotaxon 1 (1): 51-62.

Korf, R.P. \& Zhuang, W.-Y. 1985. Some new species and new records of Discomycetes in China. Mycotaxon 22: 483-514.

Korf, R.P. \& Zhuang, W.-Y. 1991. A preliminary Discomycete flora of Macaronesia: Part 11, Sarcoscyphineae. Mycotaxon 40: 1-11.

Lado, C. 2001. Nomenmyx. A nomenclatural taxabase of Myxomycetes. Cuadernos de Trabajo de Flora Micológica Ibérica 16: 1-221.

Lado, C. \& Pando, F. 1997. Myxomycetes, I. Ceratiomyxales, Echinosteliales, Liceales, Trichiales. Flora Micológica Ibérica. 2: 1-323.

Lado, C., Elisson, U., Stephenson, S.L., Estrada-Torres, A. \& Schnittler, M. 2005. (1688-1691) Proposals to conserve the names Amaurochaete against Lachnobolus, Ceratiomyxa against Famintzinia, Cribraria Pers. against Cribraria Schrad. ex J.f. Gmel. and Hemitrichia against Hyphorhamma (Myxomycetes). Taxon 54 (2): 543-545.

Larsen, M.J., Beltrán Tejera, E. \& Rodríguez-Armas, J.L. 1994. Tomentella oligofibula sp. nov. (Aphyllophorales, Thelephoraceae, s.str.) from the Canary Islands. Mycotaxon 52(1): 109-112.

Lizoñ, P. \& Korf, R.P. 1995. Taxonomy and nomenclature of Bisporella claroflava (Leotiaceae). Mycotaxon 54: 471-478.

Martin, G.W. \& Alexopoulos, C. J. 1969. The Myxomycetes. Univ. Iowa Press. Iowa. $651 \mathrm{pp}$.

Mohr, P. 2007. Drei interessante Schirmlinge (Leucocoprineae) von den Kanarischen Inseln (Spnien). Boletus 30(1): 1-11.

Nannenga-Bremekamp, N.E. 1991. A Guide to Temperate Myxomycetes. An English Translation of the Nederlandse Myxomycetes, by A. Feest \& Y. Burggraaf. Bristol, Biopress Ltd., Bristol. 409 pp.

Raitviir, A. 1980. The genus Lasiobelonium. Scripta Mycological 9: 99-132.
Rodríguez-Armas, J.L. \& Beltrán Tejera, E. 1995. Contribución al estudio de los Aphyllophorales (Basidiomycotina) del monteverde de las Islas Canarias. Bibliotheca Mycologica 160: 1-456.

Rodríguez-Armas, J.L., Beltrán Tejera, E. \& Bañares, A. 1992a. Contribución al estudio de Clavariaceae y familias afines (Aphyllophorales) de las Islas Canarias. Documents mycologiques 22 (85): 21-38.

Rodríguez-Armas, J.L., Ryvarden, L., Hallenberg, N. \& Beltrán Tejera, E. 1992b. New and noteworthy species of Aphyllophorales (Basidiomycotina) from the Canary Islands. Mycotaxon 45: 433-447.

Rodríguez-Armas, J.L., Beltrán Tejera, E. \& Bañares, A. 1994. Estudio micológico de la Reserva de la Biosfera El Canal y Los Tiles (La Palma, Islas Canarias). I. Aphyllophorales (Basidiomycotina). Documents mycologiques 23 (92): 21-38.

Roll-Hansen, F. \& Roll-Hansen, H. 1979. Ascocoryne sarcoides and Ascocoryne cylichnium. Descriptions and comparison. Norwegian Journal of Botany 26: 193-206.

Sierra López, D. 2006. Contribución al estudio de los Ascomycetes bitunicados de Cataluña. Acta Botanica Barcinonensia 50: 5-434.

Spooner, B.M. 1987. Helotiales of Australasia: Geoglossaceae, Orbiliaceae, Sclerotiniaceae, Hyaloscyphaceae. Bibliotheca Mycologica 116: 1-711.

Stadler, M., Wollweber, H., Jäger, W., Briegert, M., Venturella, G. \& Castro, J.M. 2004. Cryptic species related to Daldinia concentrica and D. eschscholzii, with notes on D. bakeri. Mycological Research 108: 257-273.

Stadler, M., Fournier, J., Granmo, A. \& Beltrán Tejera, E. 2008. The "red Hypoxylons" of the temperate Northern hemisphere. North American Fungi 3 (7): 73-125.

Stephenson, S.L. 2003. Myxomycetes of New Zealand. Vol. 3. Fungal Diversity Press, Hong Kong. 238 pp.

Undagoitia Fernández, J.R. \& Picón, R.M. 2007. Citas corológicas de la base de datos de la Sociedad Micológica de Portugalete III. Zizak 4: 29-49

Urries, M.J. 1957. Hongos microscópicos de Canarias. Publicaciones Museo Canario. Las Palmas de Gran Canaria.

Wildpret de la Torre, W., Pérez de Paz, P.L., Beltrán Tejera, E. \& Santos Guerra. 1973. Contribución al estudio de los hongos superiores de la Isla de La Palma. Vieraea 2(2): 118-128.

Recibido: 17-XII-2008

Aceptado: 29-I-2009 